\title{
Genome-wide investigation of the GRAS transcription factor family in foxtail millet (Setaria italica L.)
}

\author{
Yu Fan ${ }^{1,2}$, Xiaobao Wei ${ }^{3}$, Dili Lai ${ }^{1}$, Hao Yang ${ }^{1}$, Liang Feng ${ }^{4}$, Long Li ${ }^{5}$, Kexin Niư ${ }^{5}$, Long Chen ${ }^{6}$, Dabing Xiang ${ }^{2}$, \\ Jingjun Ruan ${ }^{1}$, Jun Yan ${ }^{2^{*}}$ and Jianping Cheng ${ }^{1 *}$
}

\begin{abstract}
Background: GRAS transcription factors perform indispensable functions in various biological processes, such as plant growth, fruit development, and biotic and abiotic stress responses. The development of whole-genome sequencing has allowed the GRAS gene family to be identified and characterized in many species. However, thorough in-depth identification or systematic analysis of GRAS family genes in foxtail millet has not been conducted.

Results: In this study, 57 GRAS genes of foxtail millet (SIGRASs) were identified and renamed according to the chromosomal distribution of the SIGRAS genes. Based on the number of conserved domains and gene structure, the SiGRAS genes were divided into 13 subfamilies via phylogenetic tree analysis. The GRAS genes were unevenly distributed on nine chromosomes, and members of the same subfamily had similar gene structures and motif compositions. Genetic structure analysis showed that most SiGRAS genes lacked introns. Some SiGRAS genes were derived from gene duplication events, and segmental duplications may have contributed more to GRAS gene family expansion than tandem duplications. Quantitative polymerase chain reaction showed significant differences in the expression of SiGRAS genes in different tissues and stages of fruits development, which indicated the complexity of the physiological functions of SIGRAS. In addition, exogenous paclobutrazol treatment significantly altered the transcription levels of DELLA subfamily members, downregulated the gibberellin content, and decreased the plant height of foxtail millet, while it increased the fruit weight. In addition, SiGRAS13 and SiGRAS25 may have the potential for genetic improvement and functional gene research in foxtail millet.
\end{abstract}

Conclusions: Collectively, this study will be helpful for further analysing the biological function of SiGRAS. Our results may contribute to improving the genetic breeding of foxtail millet.

Keywords: Setaria italica, GRAS gene family, Genome-wide analysis, DELLA protein, Fruit development, Abiotic stress

\footnotetext{
*Correspondence: yanjun62@qq.com; chengjianping63@qq.com ${ }^{1}$ College of Agriculture, Guizhou University, Guiyang 550025, People's Republic of China

${ }^{2}$ School of Food and Biological engineering, Chengdu University, Chengdu 610106, People's Republic of China

Full list of author information is available at the end of the article
}

\begin{abstract}
Background
GRAS proteins belong to a family of plant-specific transcription factors and have been widely reported to exist in higher plants [1]. The GRAS family name was based on the first three members: gibberellic acid insensitive (GAI) [2], repressor of GA1-3 mutant (RGA) [3], and scarecrow $(S C R)$ [4]. GRAS proteins usually contain 400-770 amino acid residues [5]. The $\mathrm{N}$-terminal of GRAS proteins constituted an intrinsically disordered region (IDR) peculiar to plants. This domain is highly variable and can switch
\end{abstract}


between an irregular and regular structures, which may be used as a molecular bait to bind to different target proteins. GRAS proteins involved in different signal transduction pathways and a variety of functions, thereby determining the specificity of their functions [5]. Differentiation at the N-terminal further leads to the diversification of GRAS proteins, for example, the DELLA proteins are characterized by the DELLA domain at the $\mathrm{N}$-terminal [6]. Meanwhile, the C-terminal sequence of GRAS transcription factors is relatively conserved, has specific transcription regulatory domains that bind to DNA or other proteins, and can recognize a variety of different target receptors. Thus, the C-terminal sequence participates in a variety of signal transduction regulated transcription processes [2-4]. The C-terminal of GRAS protein contains five highly conserved domains:leucineheptad repeat I (LHR I), Val-His-Ile-Ile-Asp (VHIID), leucine-heptad repeat II (LHR II), Pro-Phe-Tyr-Arg-Glu (PFYRE), and Ser-Ala-Trp (SAW) [7]. Of these domains, the conserved structural domains of GRAS proteins, the VHIID region can be used as the core region and exists in almost all GRAS proteins: V, I, H, and D represent valine, isoleucine, histidine, and aspartic acid respectively $[7,8]$. The two leucine-rich regions do not have the phenomenon of seven duplicated leucine residues that form the leucine zipper $[5,9,10]$. The posterior segment of the LHR II domain contains Leucine-X-X-LeucineLeucine (LXXLL, where $X$ stands for any amino acid) structure and is conserved in most GRAS proteins [11]. Although the functions of the PFYRE and SAW regions have not yet been elucidated, their high conservatism is suggested to be closely related to GRAS protein function [12]. The PFYRE domain shows collinearity and high similarity in all proteins, usually consisting of three components: proline residues $(\mathrm{P})$, phenylalanine and tyrosine residues (FY), arginine and glutamic acid residues (RE), and other structural domains $[11,13,14]$. The SAW motif comprises three consecutive parts near the $\mathrm{C}$ terminal, namely WX7G (X7 represents any seven amino acids), L-W, and SAW. These conserved elements play an important role in maintaining the integrity of the GRAS domain $[13,14]$.

Tian et al. [15] have systematically analyzed the GRAS family genes in Arabidopsis thaliana and rice. According to phylogenetic analysis, 57 GRAS genes in rice and 33 in Arabidopsis were divided into eight branches (DELLA, PAT1, LISCL, SCL3, SCR, LS, SHR, and HAM), which were named according to the representative genes in these subfamilies. In 2017, Cenci et al. [16] analyzed GRAS gene family members in different angiosperms, providing a clear basis for their classification. In angiosperms, the GRAS gene family not only includes the homologous members of the eight Arabidopsis GRAS subfamilies but also includes those of many other subfamilies (e.g., the NSF1, NSP2, and DLT subfamilies). GRAS proteins not only different in terms of their structure but also play a variety of physiological functions in plant growth [17]. For example, SCR is involved in cell division in the cortex and endodermis of roots in Arabidopsis, while SHR regulates the asymmetric division of roots by activating SCR proteins $[4,18,19]$. The interaction between SHR-SCR heterodimer and BIRD/ IDD transcription factors was a typical example of target-effector proteins recognition by GRAS proteins [20]. NSP1 and NSP2 can form homodimer or heterodimer, which are necessary for nodular development in legumes [21]. AtLAS regulates lateral bud formation during vegetative growth in A. thaliana [22]. LeLs (Lycopersicon esculentum lateral suppressor), a member of the AtLAS subfamily in tomato, is mainly responsible for stimulating lateral meristem development during the vegetative growth stage to promote lateral bud formation [23, 24]. OsMOC1, a homologous protein of the AtLAS subfamily in rice, plays an important role in lateral meristem development and tiller bud formation [25]. PAT1 and SCL13 of the PAT1 branch act as intermediates in the photosensitive pigment signaling pathway [24, 26]. Furthermore, AtSCL3 protein is mainly expressed in the endodermis of roots and located in the nucleus of root cells as a positive regulator of gibberellin (GA) signal transduction $[18,19]$. AtSCL3 and DELLA proteins play antagonistic roles in regulating the downstream GA response and GA homeostasis. DELLA protein exists in the GA signal transduction pathway as a negative regulator. When the GA signal recognition region on DELLA protein receives the signal, the protein degrades rapidly in the nucleus, and the plant shows a normal GA response program. Meanwhile, DELLA-gene-mutant plants will show dwarfization and a GA-insensitive phenotype [27]. In addition, DELLA protein can regulate reproductive organ development and promote fruit growth of Arabidopsis by affecting the fertilization process [28]. PhHAM of petunia is mainly expressed in the lateral organ primordium and the vascular tissue of the stem primordium. PhHAM acts on adjacent tissues in a noncellular autonomous manner to maintain the activity of the meristem of the shoot tip. The number of leaves in the petunia mutant (ham) is less than that in the wild type. Furthermore, the stem apical meristem loses its undifferentiated characteristics and forms a differentiated epidermis through the trichome, thereby preventing further organ formation [29]. The LISCL protein is regarded as the transcriptional activator of some meiosis-related genes, regulating anther microspore formation [30]. OsDLT regulates shoot and primary root development in rice, showing a dwarf phenomenon that is insensitive to brassinolide [31]. In addition, GRAS 
family members are also involved in plant responses to multiple environmental stresses. For example, the PeSCL7 in poplar can be induced by drought and highsalt stresses [32]. Salt stress, cold stress, and osmotic stress can reduce active GA content in plants, which can cause DELLA protein accumulation, inhibit plant growth, and enhance resistance to environmental stresses $[33,34]$.

Foxtail millet (Setaria italica) is an annual diploid C4 crop belonging to the Gramineae family. It has a short growth cycle and is warming-loving and reduced-sunshine and drought-tolerant [35]. Foxtail millet originated in China and is widely cultivated in arid and semiarid regions of the world as food and fodder [36]. The GRAS gene family was identified in model organisms $A$. thaliana and rice $[15,17]$, and it has been gradually identified and analyzed at the genome-wide level in more and more species, such as Solanum lycopersicum [37], Vitis vinifera [38], Malus domestica [39], Zea mays [40], Gossypium hirsutum [41], Fagopyrum tataricum [42], and Sorghum bicolor [43] and others. As a typical C4 plant, foxtail millet has not been studied for the identification and candidate gene screening of the GRAS family. In this study, 57 GRAS genes in foxtail millet were analyzed, and they were divided into 13 groups. In addition, the exonintron structure, motif composition, gene replication, chromosome distribution, and phylogenetic relationships were further analyzed. The expression of SiGRAS family members under different biological processes and abiotic stresses was also assessed.

\section{Results}

\section{Identification of GRAS genes in S. italica}

In this study, two BLAST methods were used to identify all possible GRAS members in the $S$. italica genome (Table S1). According to their location on chromosomes, the SiGRAS members were renamed SiGRASO1 to SiGRAS57. The basic characteristics that were analyzed, which included molecular weight (MW), isoelectric point (pI), coding sequence length (CDS), and subcellular localization (http://cello.life. nctu.edu.tw/).

Of the $57 \mathrm{SiGRAS}$ proteins, SiGRAS19 was the smallest with 248 amino acids, and the largest was SiGRAS26 with 912 amino acids. The molecular masses of the proteins ranged from $27.70 \mathrm{kDa}$ (SiGRAS20) to $100.09 \mathrm{kDa}$ (SiGRAS26) and the pI ranged from 4.85 (SiGRAS16) to 9.53 (SiGRAS03), with a mean of 6.35 . In the predicted subcellular localization results, 26 SiGRAS proteins were located in the nucleus, 16 were located in the cytoplasm, 13 were located in the chloroplasts, 1 was located in the chloroplasts, and 1 was located in the mitochondria (Table S1). The number of GRAS genes in S. italica was higher than that in A. thaliana (32) [15], Cucumis sativus
(37) [44], Vitis vinifera (52) [38], and Tartary buckwheat (47) [42], and lower than that in Sorghum bicolor (81) [43], Populus trichocarpa (102) [45], and Malus x domestica (127) [39].

\section{Multiple sequence alignment, phylogenetic analysis, and classification of SiGRAS genes}

To investigate the phylogenetic relationship of GRAS proteins in the foxtail millet, we constructed a phylogenetic tree based on the amino acid sequences of the 57 identified SiGRAS, 33 AtGRAS, and 50 OsGRAS proteins (Fig. 1, Tables S1 and S2). According to the previously proposed classification method and topological structure proposed by Cenci and Rouard [16], the 140 GRAS proteins in the phylogenetic tree were divided into 13 main clades. This finding was consistent with the previous classification of the GRAS taxonomic subfamilies in angiosperms. These data indicate that these proteins had no loss during the evolution of S. italica. These subfamilies of GRAS proteins widely existing in different angiosperms may play a basic role in plant development and evolution, similar to those recently reported in other plant species, including Amborella trichopoda, Phoenix dactylifera, $V$. vinifera, Musa acuminata, O. sativa, A. thaliana, Theobroma cacao and Coffea canephora [16]. Among the 13 subfamilies, LISCL had the most members (18 SiGRAS proteins), and DLT (SiGRAS23), SCL4/7(SiGRAS47), OS19(SiGRAS30), OS4(SiGRAS28), and OS43(SiGRAS42) had the fewest (only one SiGRAS protein) (Fig. 1, Tables S1 and S2). There were eight, seven, six, six, three, two, and two SiGRAS proteins in the PAT1, SCL3, SHR, HAM, DELLA, SCR, and LAS subfamilies, respectively. Some SiGRAS proteins were tightly grouped with the AtGRAS and OsGRAS proteins (bootstrap support $\geq 70$ ). These proteins may be orthologous to AtGRASs or OsGRASs, and may have similar physiological functions.

These GRAS proteins of Arabidopsis and rice, which have high homology and similar protein structures to SiGRASs, were selected for multiple sequence alignment. Furthermore, the LHR I, VHIID, LHR II, PFYRE, and SAW domains of these GRAS proteins were further compared. As shown in Fig. S1, the VHIID domain is considered to be the core region, which contained a characteristic amino acid sequence. Although its amino acid structure was highly similar in different plant species, this region was not completely conserved. Further, the His and Asp amino acid residues in the domain were more conserved, and these residues may be necessary for the function of GRAS proteins in different subfamilies [15-17]. Similar to the GRAS proteins of sorghum [43] and rice [15], the N-terminal of SiGRAS proteins 


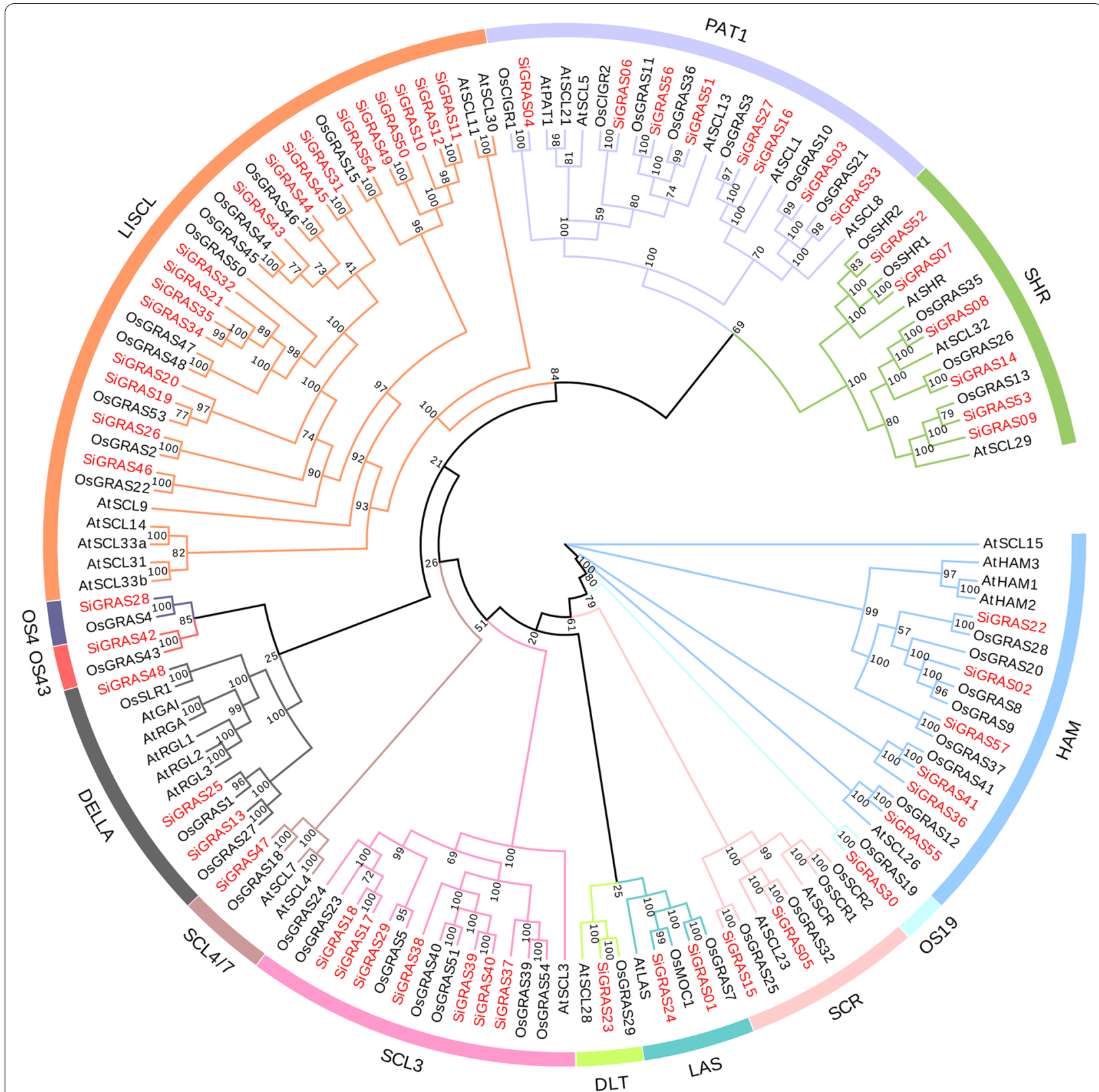

Fig. 1 Unrooted phylogenetic tree representing relationships among GRAS domains of S. italica, Arabidopsis, and rice. The phylogenetic trees were derived using the NJ method in MEGA7.0. The tree shows the 13 phylogenetic subfamilies marked with the red font on a white background. GRAS proteins from Arabidopsis and O. sativa have the prefix 'At' and 'Os', respectively

contains a highly disordered region, and shows certain similarities among different subfamilies [5].

\section{Conserved motifs, gene structures, and cis-acting elements analysis of SiGRAS genes}

By comparing the genomic DNA sequences of SiGRAS genes, we obtained the intron and exon structures of SiGRAS genes to further understand the structural composition of these genes (Fig. 2, Tables S1 and S3). A comparison of the localization and number of the exonintron structures revealed that the 57 SiGRAS genes had different numbers of exons, varying from 1 to 5 (Fig. 2A, B). The 57 SiGRAS genes all contained the GRAS domain, and most of them $(37, \sim 64.91 \%)$ contained no introns; 13 SiGRAS genes contained one intron; SiGRASO4, SiGRAS12, SiGRAS16, SiGRAS27, SiGRAS38, and 


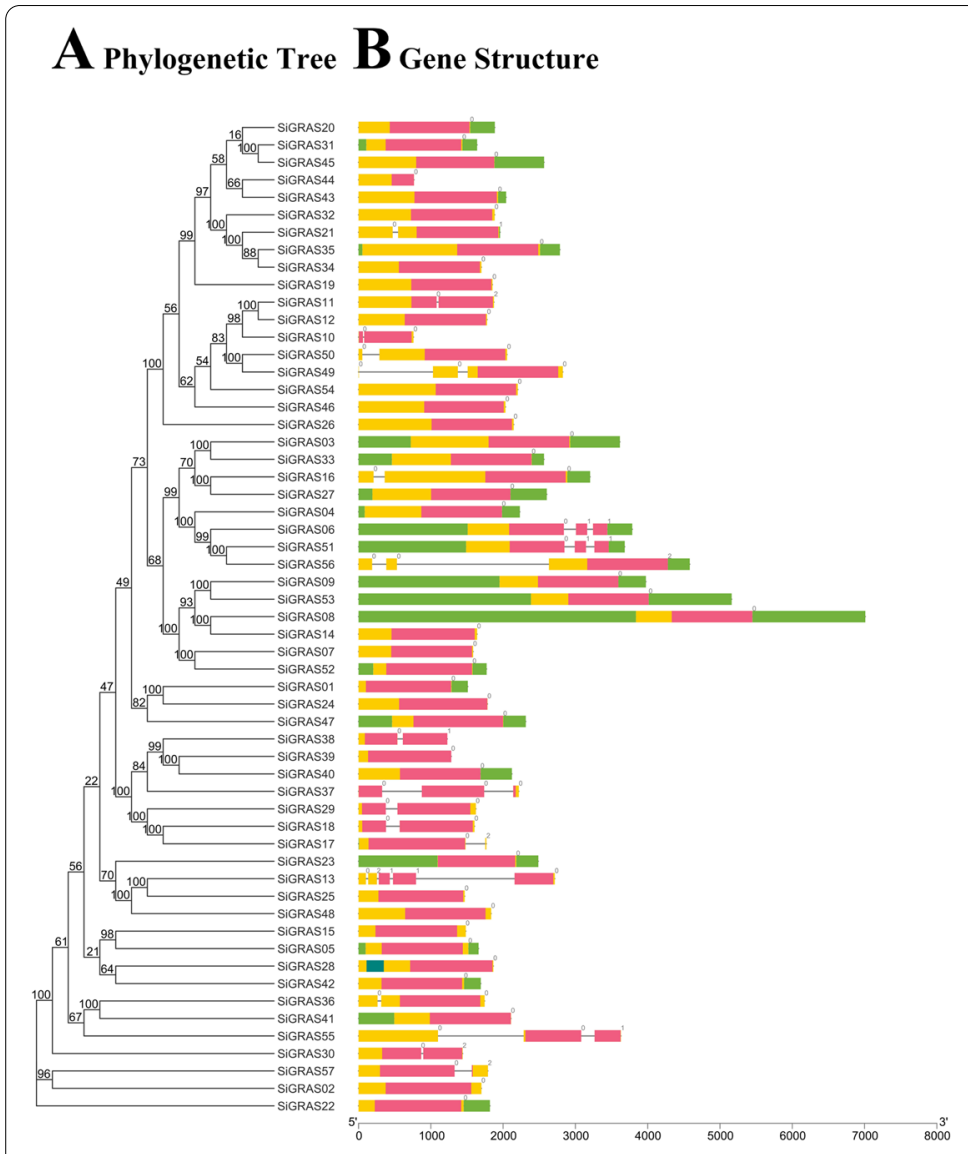

\section{Motif Pattern}

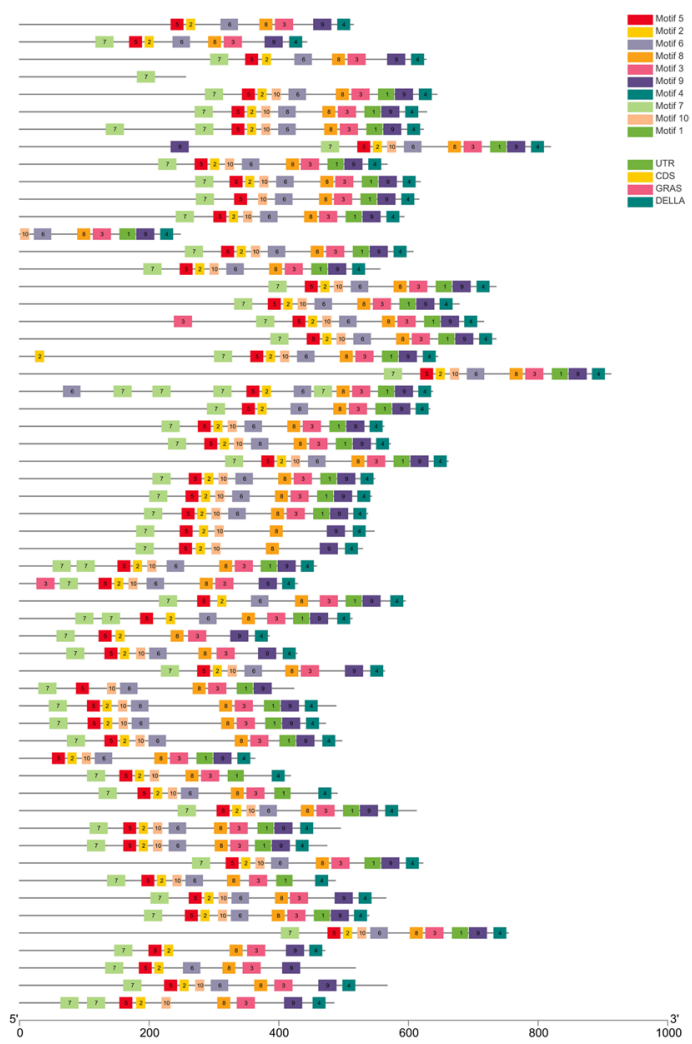

Fig. 2 Phylogenetic relationship, gene-structure analysis, and motif distributions of S. italica GRAS genes. A Phylogenetic tree was constructed by the NJ method with 1000 replicates on each node. B Exons and introns are indicated by rectangles and gray lines, respectively. These numbers are generated based on the "phase" of the annotated file, which is about the different phases of CDS in the gene. Meanwhile, "phase" is defined as "0", "1", and "2". C Amino acid motifs in the S. italica GRAS proteins (1-10) are represented by colored boxes. The black lines indicate relative protein lengths

SiGRAS42 contained two introns; and SiGRAS18 had four introns, which was the highest number of introns. The 37 genes without introns were distributed across the other 12 subfamilies, except for the Os43 subfamily, and were mainly part of the LISCL subfamily. In general, members of the same subfamily had similar gene structures. Members of the DELLA, DLT, HAM, LAS, OS19, OS4, SCL4/7, SCR, and SHR subfamilies contained no introns or one intron. Further analyses indicated that the SCL3 subfamily was the most diverse in terms of the number of introns.

To further study the characteristic regions of the SiGRAS proteins, their motifs were analyzed using an online MEME. A total of 10 distinct conserved motifs (named motifs 1-10) were found (Fig. 2C, Table S3). As exhibited in Fig. 2C, motif 8, 3, 9, and 4 were widely distributed in the SiGRAS family, except for SiGRAS41. SiGRAS members of the same subfamily usually shared a similar motif composition. For example, the DELLA subfamily contained motifs $7,5,2,10,6,8,3,1,9$, and
4; the LISCL subfamily contained motifs $6,8,3$, 9, and 4; and the HAM subfamily contained motifs 5, 2, 8, 3, and 9 . Some motifs were only distributed in specific locations of the pattern. For example, the motif $7(50, \sim 87.72 \%)$ was always distributed at the start of the pattern, and motif $4(55, \sim 96.49 \%)$ was almost always at the end of the pattern. The functions of most of these conserved motifs remain to be elucidated.

The cis-acting elements in the promoter regions (2000 bp) of 57 SiGRAS genes were further investigated. A total of 110 cis-regulatory elements were identified (Table S4), which could be divided into seven categories: development-related, environmental stress-related, hormone-responsive, light-responsive, promoter-related, site binding-related, and others. Among them, the lightresponse elements accounted for a large proportion, including 30 cis-regulatory factors. The promoter related element (CAAT-box, TATA-box elements) and other elements (MYB, MYC, and the unnamed 4 elements) in the promoter region were identified in all SiGRAS genes 
(57 members). There were ten hormone-responsive elements in the 57 SiGRAS genes of the foxtail millet, which covered most plant hormones, including abscisic acid responsive (ABRE), auxin responsive (AuxRR-core, TGAelement), gibberellin-responsive (GARE-motif, P-box, TATC-box), jasmonic acid-responsive (CGTCA-motif, TGACG-motif), and salicylic acid-responsive (TCAelement) elements. In addition, cis-regulatory elements related to low-temperature, anoxia, drought, anaerobic conditions, other defenses, and stress responses were found in SiGRAS genes. Nearly $85 \%$ of SiGRAS genes contained anaerobic induction-responsive elements, light-responsive elements, abscisic acid-responsive elements, and MeJA responsive-responsive elements; whereas only approximately $28 \%$ of GRAS genes contained GA--responsive and auxin-responsive elements. Some cis-acting elements may regulate the expression of different tissues (seed, root, endosperm, palisade mesophyll, and meristem) during development. It can be inferred that SiGRAS genes can not only participate in the tissue development process but also respond to a variety of abiotic stresses.

\section{Chromosomal spread and gene duplication in SiGRAS genes}

The name of each SiGRAS gene name corresponds to its physical position from the top to the bottom of chromosomes 1 (Chr I) to 9 (Chr IX) of S. italica (Fig. 3, Tables S1 and S5). The distribution of the 57 SiGRAS genes on the chromosomes was uneven. Interestingly, SiGRAS genes were not found on Chr6 (Chr VI). Chr3 and Chr9 contained the largest number of SiGRAS genes (12 genes, $\sim 21.05 \%$ ), followed by Chr7 (10, $\sim 17.54 \%)$; Chr1 and Chr4 contained the fewest SiGRAS genes (three each, 5.26\%). Chr5, Chr2, and Chr8 contained five $(\sim 8.77 \%)$, six $(\sim 10.53 \%)$, and six $(\sim 10.53 \%)$ SiGRAS genes, respectively. A chromosomal region within $200 \mathrm{~kb}$ range containing two or more genes was defined as a tandem duplication event [46]. On Chr3, 7, 8, and 9, we found eight tandem duplication events involving 13 SiGRAS genes (Fig. 3). SiGRAS11, SiGRAS38 and SiGRAS44 each had two tandem repeat events (SiGRAS11 and SiGRAS10/SiGRAS12; SiGRAS38 and SiGRAS37/SiGRAS39; SiGRAS44 and SiGRAS43/SiGRAS45). All SiGRAS genes that formed tandem repeat events belonged to the same subfamily. For example, SiGRAS49 and SiGRAS50 were tandem repeat genes that were clustered together in the LISCL subfamily (Fig. 3, Table S1). Ten of the thirteen tandem repeat genes were derived from the LISCL subfamily, which suggested that it played a major role in GRAS genes expansion in evolution. Furthermore, the LISCL subfamily is also the subfamily with the highest

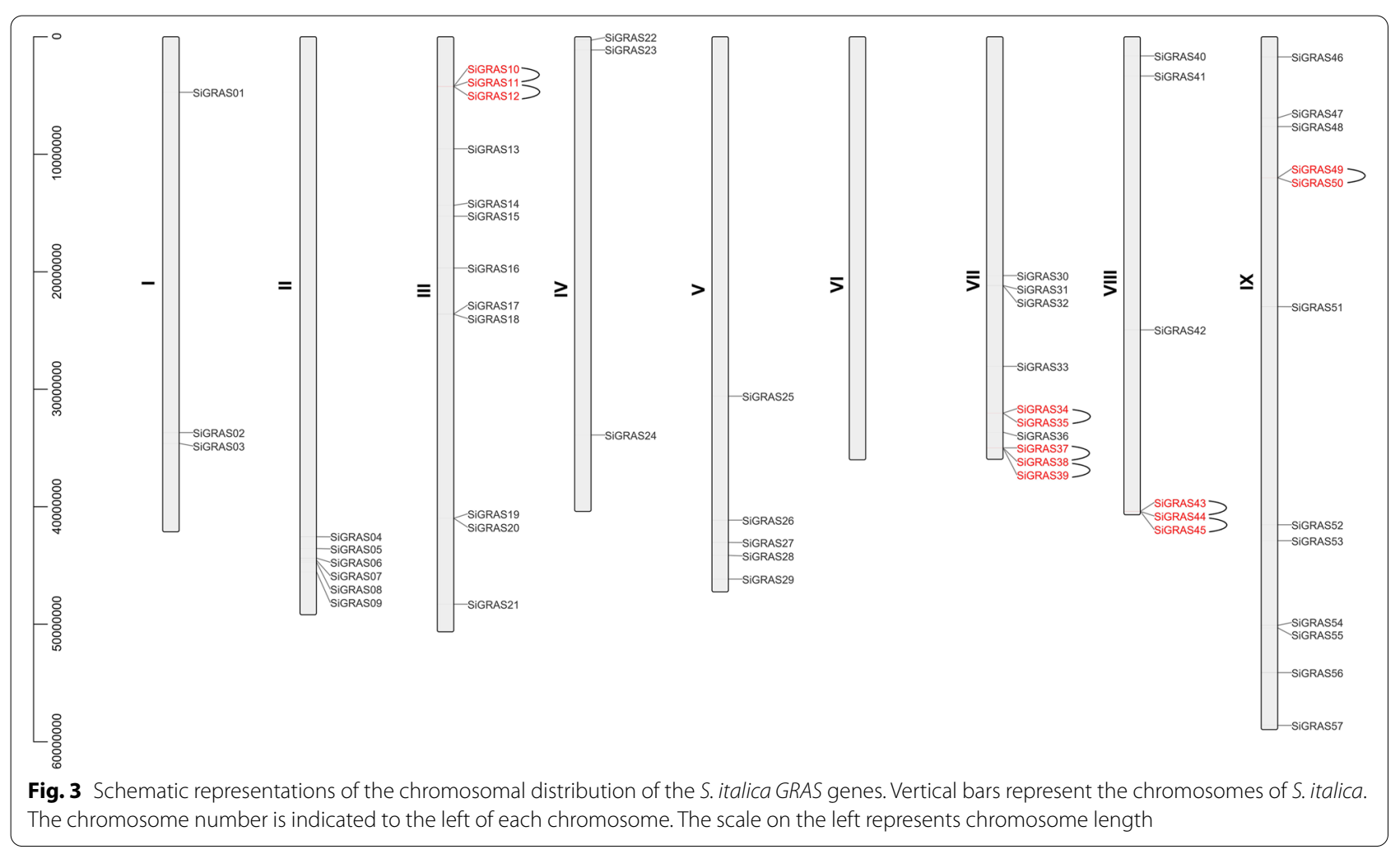


number of members. Only SiGRAS37, SiGRAS38 ${ }_{2}$ and SiGRAS39 were from the SCL3 subfamily.

In addition, there were 12 pairs of segmental duplications in the SiGRAS genes (Fig. 4, Table S6). As shown in Fig. 5, $19(\sim 33.33 \%)$ paralogs were identified in the SiGRAS gene family, which indicated an evolutionary relationship for these GRAS genes. Although located on different chromosomes, SiGRAS46/SiGRAS54 is closely related to SiGRAS26, which may be evidence of a gradual expansion of the LISCL subfamily. The segmental duplications were unevenly distributed in nine linkage groups (LG) of S. italica. LG3 had four SiGRAS genes, whereas LG2 and LG5 had only one SiGRAS gene. All of the segmental repeat gene pairs came from the same subfamily, (Table S6). For example, SiGRAS46 and SiGRAS54/ SiGRAS26 were segmental paralogs clustered together

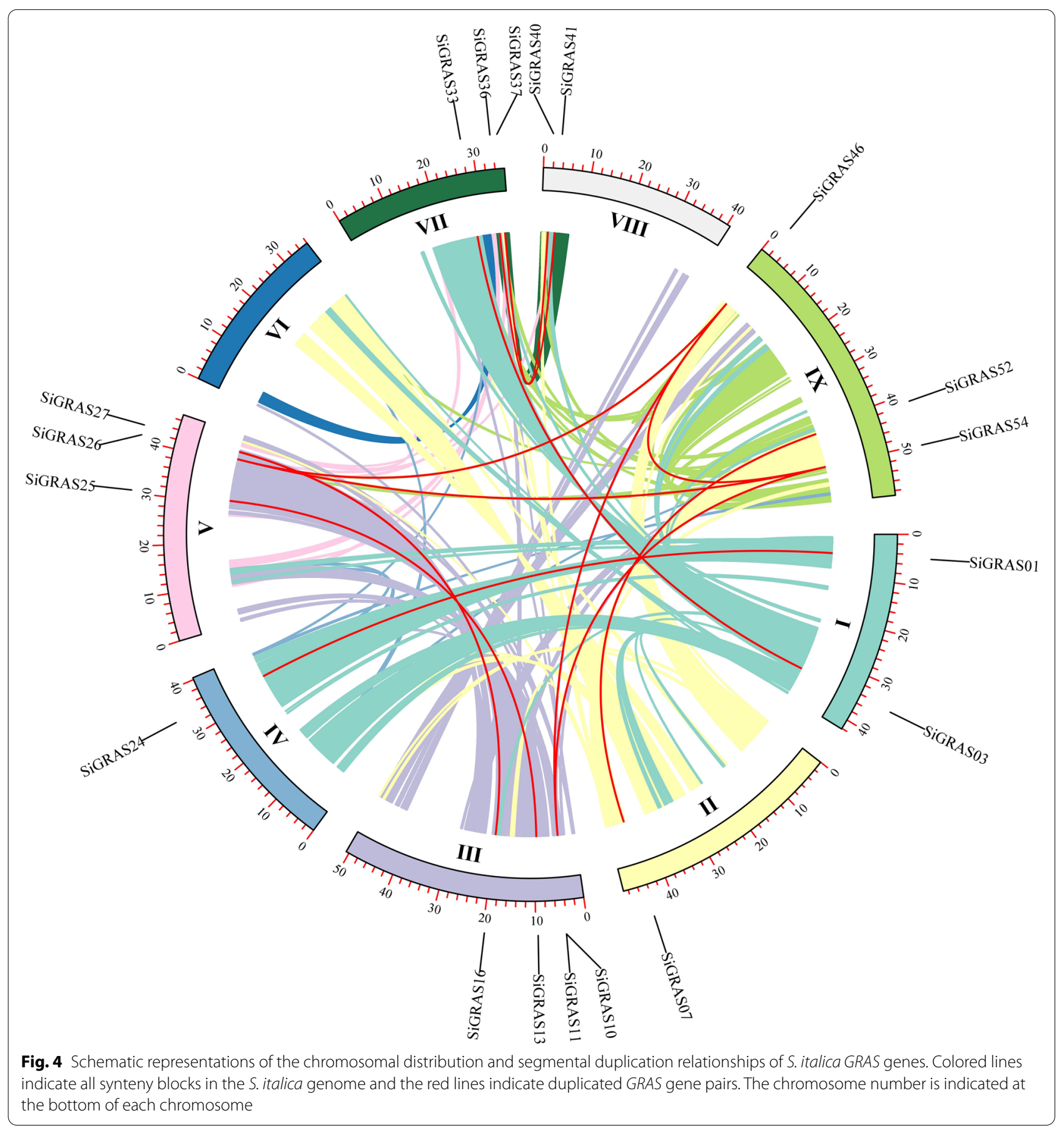




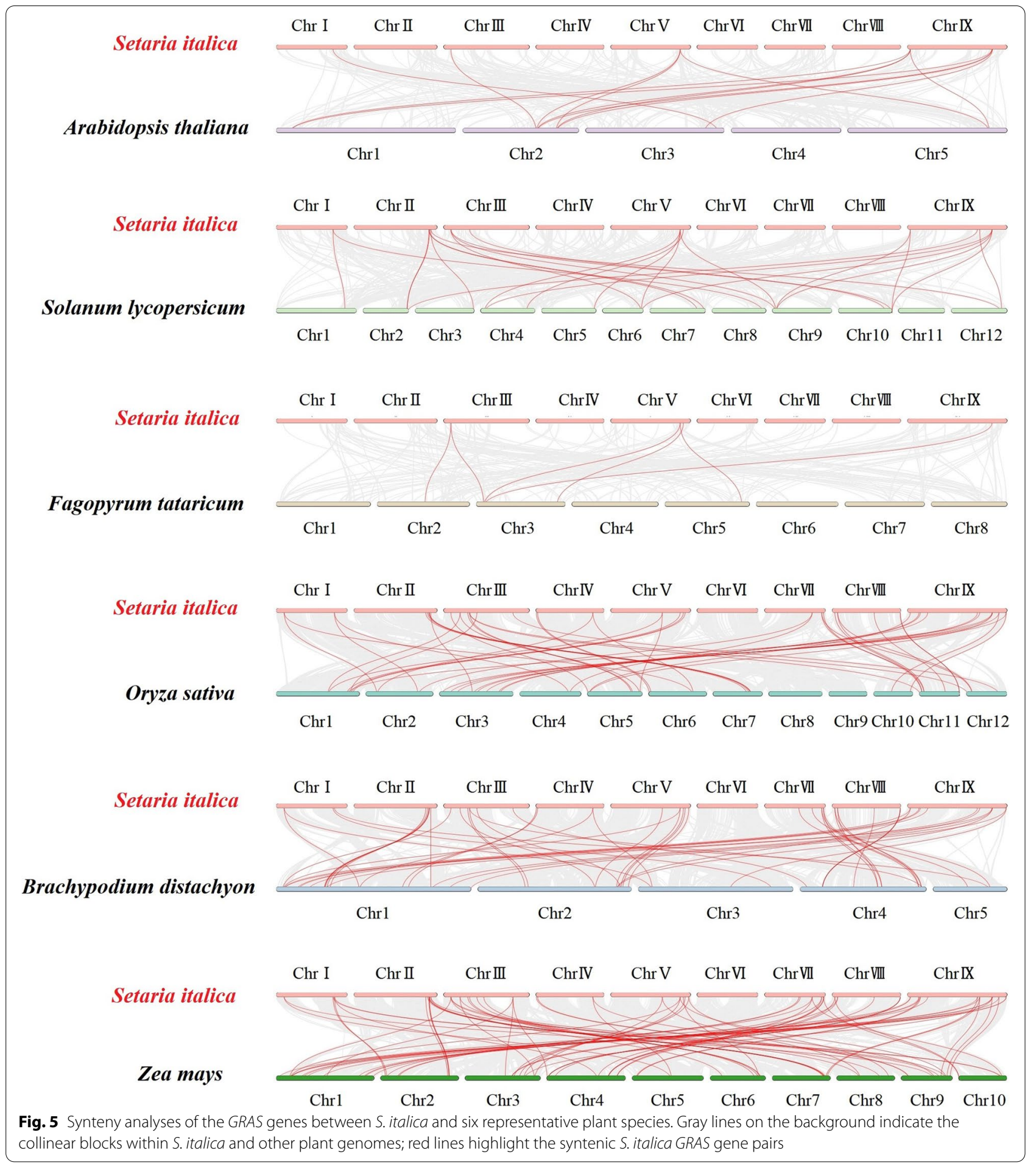

in the LISCL subfamily, which had the largest number of linked genes $(5 / 19, \sim 26.32 \%)$. In addition, the PAT1 subfamily had four segmental duplications, whereas the DELLA, HAM, LAS, SCL3, and SHR subfamilies had only one pair of segmental duplication.

\section{Synteny analysis of SiGRAS genes}

To further infer the syntenic relationships of the GRAS genes, we constructed six comparative syntenic maps of $S$. italica with six representative species: three dicotyledons (A. thaliana, S. lycopersicum and F. tataricum) 
and three monocotyledons (O. sativa, B. distachyon, and Z. mays) (Fig. 5, Table S7). We found that a total of 47 SiGRAS genes showed collinear relationships with those in Arabidopsis (6), F. tataricum (4), tomato (15), B. distachyon (37), rice (38), and maize (59). The number of orthologous gene pairs between foxtail millet and the other six species (Arabidopsis, tomato, F. tataricum, B. distachyon, rice, and maize) was $13,25,6,53,52$, and 81 , respectively.

Some SiGRAS genes were found to exist in at least one pair of collinear genes in six plants, such as SiGRAS10 with AT2G29060/Solyc10g086530/FtPinG0201759800/B GIOSGA018951/BRADI_1g03620v3/Zm00001d028603, which suggested that these orthologous genes already existed before the ancestral divergence. As expected, many orthologous gene pairs (with 12 SiGRAS genes) identified between $S$. italica and B. distachyon/rice/ maize were not identified in S. italica and Arabidopsis/ tomato/F. tataricum, such as SiGRAS13 with BGIOSG A001121/BRADI_2g45117v3/Zm00001d044065, and SiGRAS25 with BGIOSGA001121/BRADI_2g45117v3/ $Z m 00001 d 044065$. This suggests that these orthologous pairs may have been gradually formed after the independent differentiation of monocotyledons (Table S7). To better observe the evolutionary constraints of the $57 \mathrm{SiGRAS}$ genes, the SiGRAS genes were subjected to the Tajima D neutrality test $[47,48]$. The calculated D of 6.77 deviated significantly from 0 , suggesting that the SiGRAS gene family might have been involved in purification and selection pressure during the evolution process (Table S8).

\section{Evolutionary analysis of the SiGRAS and GRAS genes of several different species}

To analyze the evolutionary relationship of the trihelix family of GRAS proteins among S. italica and six plants (A. thaliana, F. tataricum, S. lycopersicum, B. distachyon, $O$. sativa, and $Z$. mays), an unrooted neighbor-joining (NJ) tree was constructed. The cluster tree contained ten conserved motifs according to the MEME web server relative to the protein sequences of the 57 identified SiGRAS genes and the GRAS genes of the six other plants (Fig. S2, Table S3).

As shown in Fig. S2, the GRAS proteins of S. italica tended to cluster with those of $O$. sativa and $Z$. mays, which suggested that these GRAS proteins were more closely related. Furthermore, some homologous GRAS proteins had similar motifs. Most GRAS proteins of these seven plants contained motifs 7, 5, 2, 6, 9, 3, and 8 . In addition, several motifs existed in specific topologies, such as motif 4 . Interestingly, some motifs tended to be located in a specific composition. For example, motif 5 was always located between motifs 7 and 2, motif 10 was always located at the start of the pattern, and motif 8 was almost always located at the end of the pattern. In general, the GRAS proteins of O. sativa, Z. mays, and S. italica on the same branch had similar motif compositions. Similar compositions tended to cluster in specific GRAS protein subfamilies, which indicated potential functional similarities among those proteins.

\section{Expression patterns of SiGRAS genes in several organs}

To investigate the potential roles of these SiGRAS genes, real-time polymerase chain reaction was used to detect the expression of 15 individual members from different subfamilies in five organs (third leaf, flag leaves, stems, roots, and fruits) (Fig. 6). The results showed that the expression patterns of SiGRAS genes varied greatly in different tissues and organs, suggesting that they have multiple functions in foxtail millet growth and development. Some genes showed preferential expression in the detected tissues. Four genes (SiGRASO1, SiGRAS05, SiGRAS13, SiGRAS30) were most highly expressed in the fruits, five genes (SiGRAS04, SiGRAS12, SiGRAS18, SiGRAS41, and SiGRAS47) were most highly expressed in the third leaves, and SiGRASO7 and SiGRAS28 were most highly expressed in the flag leaves. In addition, SiGRAS48 and SiGRAS42 showed high expression in the stems and roots, respectively.

Further, some SiGRAS genes may regulate the fruit development of foxtail millet, thus affecting its nutritional composition and development rate. Therefore, we explored to study the expression of these $15 \mathrm{SiGRAS}$ genes at 18 (early filling stage), 25 (middle filling stage), and 32 (initial maturity stage) days post-anthesis (DPA) to identify the genes that may regulate $S$. italica fruit development (Fig. S3). The results showed that the expression levels of most SiGRAS genes were different in the fruits and glume during the three stages of fruit development. SiGRAS18 expression increased with foxtail millet fruit development, whereas the expression level of SiGRAS41 expression decreased with fruit development. Interestingly, the expression level of most genes (SiGRAS01, SiGRAS05, SiGRAS07, SiGRAS13, SiGRAS23, SiGRAS25, SiGRAS30, and SiGRAS42) were the highest at 25 DPA, while the expression level of SiGRAS12 and SiGRAS47 was at its lowest level at 25 DPA. In the glume, the expression level of six genes (SiGRAS04, SiGRAS05, SiGRAS07, SiGRAS28, SiGRAS41, and SiGRAS42) decreased with fruit development, whereas the expression of four genes (SiGRAS01, SiGRAS18, SiGRAS30, and SiGRAS48) increased.

The expression patterns of SiGRAS genes were coordinated in several plant organs, which indicates that their roles may be synergistic (Fig. S4). Most SiGRAS genes showed significant positive correlations; for 

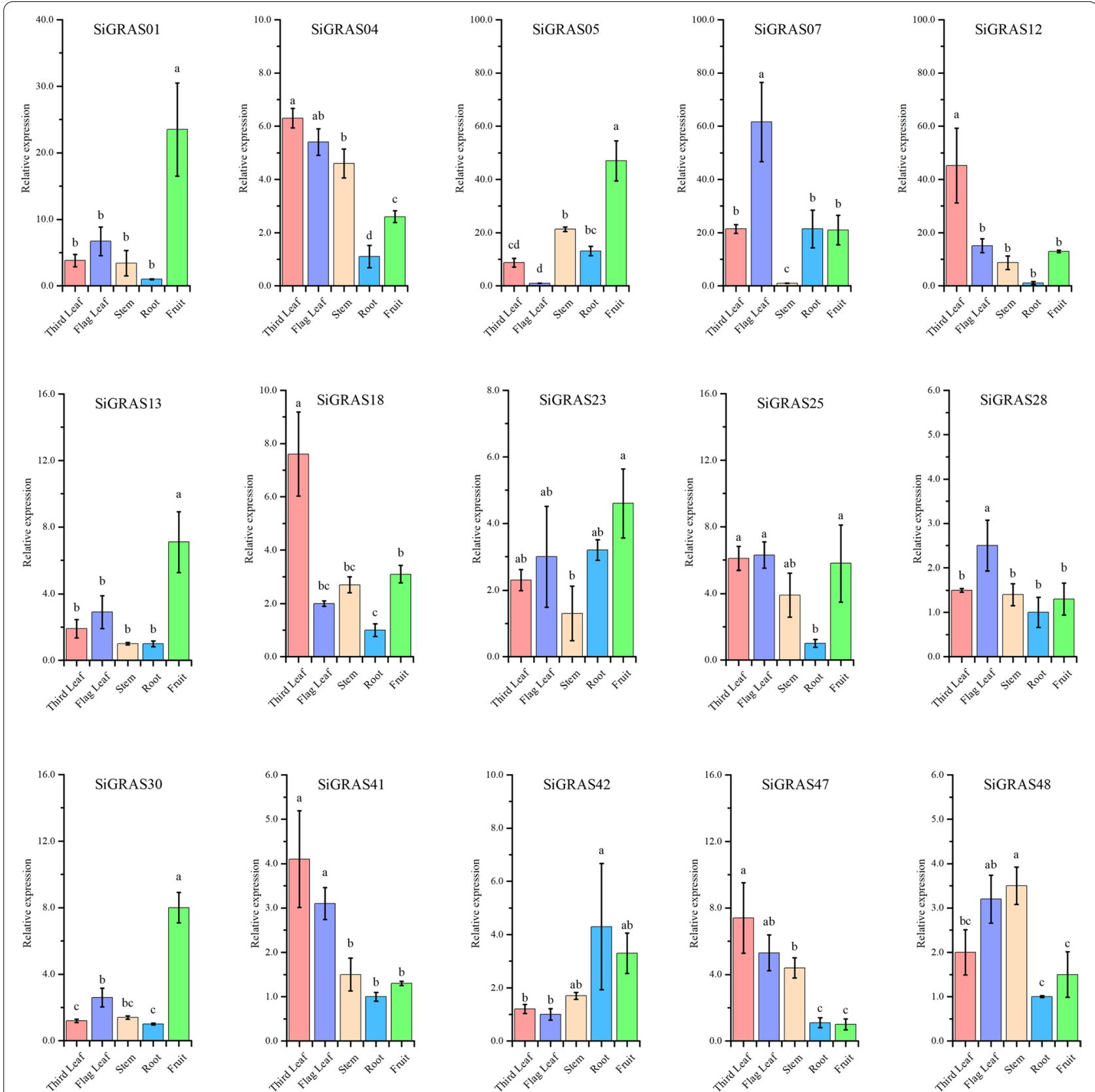

Fig. 6 Expression patterns of 15S. italica GRAS genes in the third leaf, flag leaf, root, stem, and fruit organs were examined by qRT-PCR. Detail: SiGRAS01, SiGRAS04, SiGRAS05, SiGRAS07, SiGRAS12, SiGRAS13, SiGRAS18, SiGRAS23, SiGRAS25, SiGRAS28, SiGRAS30, SiGRAS41, SiGRAS42, SiGRAS47, and SiGRAS48 are from subfamily LAS, PAT1, SCR, SHR, LISCL, DELLA, SCL3, DLT, DELLA, OS4, OS19, HAM, OS43, SCL4/7, and DELLA, respectively. As far as possible, these subfamilies have distant clustering relationships and significant differences in their amino acid structures. Error bars were obtained from three measurements. Lowercase letters above the bars indicate significant differences ( $a=0.05, L S D)$ among the treatments. The SE is selected as the value of the bar. The same is below

example, five genes (SiGRAS05, SiGRAS07, SiGRAS13, SiGRAS23, and SiGRAS25) were significantly positively correlated. SiGRASO4, SiGRAS28, and SiGRAS42 also showed a significantly positive correlation.
However, many pairs of SiGRAS genes (SiGRAS18 and SiGRAS05/SiGRAS07/SiGRAS23/SiGRAS25; SiGRAS47 and SiGRAS13/SiGRAS25; SiGRAS12 and SiGRAS23) were significantly negatively correlated. 


\section{Effects on grain development and DELLA subfamily expression of after paclobutrazol treatment}

The plant height, 1000-grain weight, and gibberellin content were observed at different stages of grain development in foxtail millet after paclobutrazol (Fig. 7A). The results showed that the plant height of foxtail millet was decreased by paclobutrazol treatment, whereas the 1000-grain weight was increased significantly, particularly in the later stage of grain development. Additionally, the endogenous GA content of both the mock and paclobutrazol treatment groups decreased during grain development. We found that the GA content of the paclobutrazol treatment group dropped to a lower level more dramatically in the early filling stage (18 DPA), but there was no significant difference at the initial maturity stage (32 DPA).

We further investigated the expression of DELLA subfamily genes (SiGRAS13, SiGRAS25, and SiGRAS48) when foxtail millet was treated with $250 \mathrm{mg} / \mathrm{L}$ exogenous paclobutrazol (Fig. 7B) [42]. The experimental group was treated with paclobutrazol, while the control group was treated with the same amount of water. The

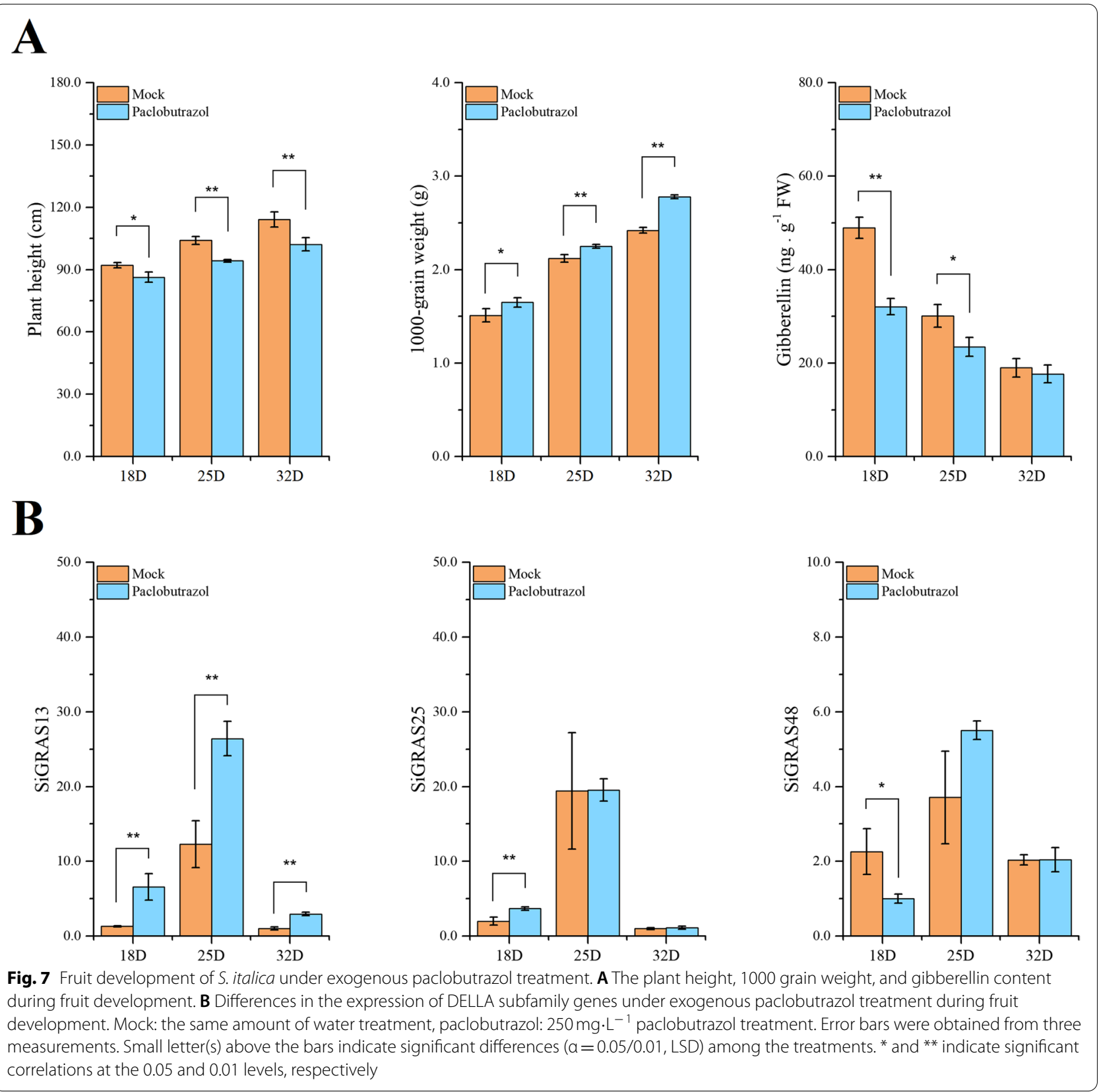


results showed that the expression patterns of the three DELLA genes were significantly altered during fruit development. The expression of all genes showed an initial increasing trend followed by a decrease, and the expression level reached the maximum value at 25 DPA. SiGRAS25 expression was increased at 18 DPA, compared with that in the control group, while SiGRAS48 expression decreased at the early filling stage. Notably, the expression of SiGRAS13 increased significantly during the whole grain development stage, and its response to external paclobutrazol was more significant.

\section{Expression patterns of SiGRAS genes in response to different abiotic stresses}

To further determine whether the expression of SiGRAS genes is influenced by different abiotic stresses, 15 SiGRAS genes were examined for their expression under eight abiotic stresses: acid ( $\mathrm{HCl} 0.1 \mathrm{~mol} / \mathrm{L})$, alkali $(0.2 \mathrm{~mol} / \mathrm{L})$, polyethylene glycol (PEG, $10 \%), \mathrm{NaCl}(5 \%)$, heat $\left(40^{\circ} \mathrm{C}\right)$, cold $\left(4{ }^{\circ} \mathrm{C}\right)$, flooding and darkness. Overall, many SiGRAS genes were significantly induced/ repressed by the different forms of stress (Fig. S5). The expression levels of SiGRAS genes changed with time or in different organs, depending on the specific treatments. For example, under heat stress, the expression of SiGRAS04, SiGRAS05, SiGRAS07, SiGRAS12, SiGRAS13, SiGRAS23, SiGRAS25, and SiGRAS47 were first significantly upregulated in the roots and stems but was then downregulated. Under cold stress, SiGRAS18 expression was significantly downregulated in the stems and leaves at $24 \mathrm{~h}$, whereas it was significantly up-regulated in the roots. Under $\mathrm{NaCl}$ stress, SiGRAS41 expression was significantly upregulated in the roots, but downregulated in stems. Interestingly, several SiGRAS members showed opposing expression patterns under different treatments. The expression level of SiGRAS41 was upregulated in stems and leaves in heat treatment, whereas its expression pattern was reversed by cold stress. The expression of some genes showed similar patterns under different stress treatments. For example, SiGRAS04 expression was initially unchanged but was then significantly upregulated in the roots, stems, and leaves by the heat and cold treatments. Some other genes showed changes in specific organs. For instance, SiGRAS23 responded significantly to heat and cold treatment in the leaves and roots, and SiGRAS42 responded significantly to acid and alkali treatment in the roots $(P<0.05)$. In most treatments, the expression of SiGRAS13 (DELLA) increased significantly. Furthermore, correlations between SiGRAS gene expression patterns were observed (Fig. S6). Most of the SiGRAS members were weakly related. However, a few SiGRAS genes were significantly positively correlated, such as SiGRAS05 with SiGRASO7, and SiGRAS28 with SiGRAS48 $(P<0.05)$.

\section{Discussion \\ SiGRAS gene structure and evolutionary analysis}

The GRAS gene family of millet was systematically analyzed, and a total of 57 SiGRAS genes were identified. All the proteins showed significant structural differences, which indicated the high complexity of the GRAS family. The length of GRAS protein ranges from 248 to 912 amino acids, which account for the length and sequence variability of GRAS [6-9]. The ratio of SiGRAS genes to the total number of genes in the $S$. italica genome $(\sim 38,801$ genes) $[49,50]$ was approximately $0.17 \%$, which was more than that in Arabidopsis (0.11\%) [15], rice $(0.15 \%)$ [17], tomato $(0.15 \%)$ [37], C. sativus $(0.14 \%)$ [44] and Tartary buckwheat (0.14\%) [42], but less than in Carica papaya (0.31\%) [45] and Medicago truncatula $(0.29 \%)$ [12]. The GRAS proteins of millet were divided into 13 subfamilies in the phylogenetic analysis, including LISCL, SCL4/7, DELLA, HAM, SHR, PAT1, SCR, OS4, SCL3, LAS, OS19, and DLT (Fig. 1). At least one SiGRAS protein has been identified in each Arabidopsis subpopulation, suggesting that these GRAS subfamilies have not been lost during long-term evolution and may play some fundamental biological functions $[15,17]$. Our study supports that the hypothesis that the separation of the GRAS family may precede the separation of millet and $A$. thaliana [15]. As expected, some SiGRAS proteins (SiGRAS28, SiGRAS30, and SiGRAS42) are classified into rice specific subfamilies, which suggested that further differentiation of the GRAS family in monocotyledons may result in the formation of independent branches $[15,17]$. As such, the unique physiological functions of these SiGRAS proteins need further study. Among the 13 subfamilies, the LISCL subfamily has the highest number of members $(18, \sim 31.6 \%)$. Meanwhile, the DLT (SIGRAS23), SCL4/7 (SIGRAS47), OS19 (SIGRAS30), OS4 (SIGRAS28) and OS43 (SIGRAS42) subfamilies had the fewest members (only one SiGRAS). Further expansion of the LISCL subfamily is supported by high homology among members (SiGRAS26, SiGRAS46, and SiGRAS54) of several different chromosomes. Similar to the GRAS subfamilies of other plants, such as A. thaliana [15], rice [17], buckwheat [42], and sorghum [43], the different subfamilies may have different differentiation abilities in the long-term evolutionary process. Evolutionary differences in these genes may be important for their various functions in species. However, there is insufficient research to show that the process of environmental adaptation is related to differences in differentiation among subfamilies. 
The highly variable $\mathrm{N}$-terminus of GRAS protein constitutes IDRs, which contain molecular recognition features and some easily exchanged gene fragments. These functional regions can complete the recognition of their specific binding objects through disordered and ordered transformation [1,51]. SiGRAS proteins showed abundant $\mathrm{N}$-terminal differences, which indicated their functional diversity of SiGRAS proteins (Fig. S1). The VHIID region of all subfamilies except the LISCL subfamily is relatively conserved, especially in the histidine " $\mathrm{H}$ " and aspartic acid "D" of amino acid residues. Meanwhile, the relative variability of valine "V" and isoleucine "I" was speculated to be caused by gene mutations $[52,53]$. Nevertheless, the same subfamilies have similar amino acid structures, and they may perform similar physiological functions [42]. In addition, some GRAS proteins (SigRAS31 SigRAS32, SiGRAS43, SiGRAS45), which were attributed to the LISCL subfamily, did not have conserved the histidine " $\mathrm{H}$ " and aspartic acid " $\mathrm{D}$ " in the VHIID region. This discrepancy may be evidence of further differentiation of the GRAS genes, with new structures giving rise to new functions. A similar phenomenon has been found in sorghum [43], which is different from that in Arabidopsis [15]. We speculate that the high activity of the LISCL subfamily leads to structural differentiation, which may be due to the instability of amino acids. This phenomenon may be the reason for the expansion of the subfamily, leading it to become the largest subfamily. Further, the SiPAT1 and SiSCR subfamilies have the most conserved structures, which are similar in the lower mosses and ferns and the higher species [54]. We also observed some domain loss events in SiGRAS19 and SiGRAS20 (Fig. S1), both of which were classified into the LISCL subfamily, which may be the result of heterotopic or inversion of chromosome fragments [55]. Domain gain and loss is a driving force for gene family expansion, which often occurs in monocotyledonous plants such as rice and maize $[56,57]$. The proportion of SiGRAS genes without introns $(37, \sim 64.9 \%)$ was higher than that of rice (55\%) [15] and poplar (54.7\%) [32], lower than that of Tartary buckwheat $(41, \sim 87 \%)$ [42] and close to that of sorghum (66.67\%) [43]. Genes without introns are also found in other large gene families, such as the F-box transcription factor gene family [58] and DEAD-box RNA helicase [59]. GRAS genes in plants may originate from prokaryotic genes through horizontal gene transfer and repeated events during evolution; as such, a large number of GRAS gene family members are intron-free genes [41]. From the perspective of evolution, the existence of introns can increase the length of genes and the frequency of intergene recombination, thereby conferring a positive effect on evolution [60]. However, intronfree genes are not isolated during transcription and translation, can continuously encode proteins, and tend to respond quickly to environmental changes [61, 62].

The tandem repetitions and fragment replication events play key roles in the expansion of GRAS gene family in foxtail millet. The proportion of GRAS protein in foxtail millet is higher than that in A. thaliana and rice $[15](\sim 0.17 \%)$, which indicates that there may be more gene repetition events or higher retention frequency after gene replication in millet. We found eight tandem repeat events in the SiGRAS gene family involving 13 members $(\sim 22.8 \%)$, which is higher than that in Arabidopsis (2/34) [15], plum (10/45) [63], and tomato (15/53) [37], but lower than that in poplar (40/106) [32] and sorghum (25/81) [43]. Notably, most tandem duplicates of SiGRAS genes were from the same subfamily, and mainly occurred in the LISCL subfamily $(10, \sim 76.9 \%)$. This indicates that members of some GRAS protein subfamilies have a higher degree of preference for replication events, and these genes do not show great structural differences after replication events [43]. In addition, this study found that fragment duplication (29 SiGRAS genes, 33.3\%) contributed slightly more to the increase of GRAS members in millet than tandem duplication, which was similar to that in tartary buckwheat [42] and sorghum [43]. This may indicate that the LISCL subfamily may have a stronger expansion in plant evolution, not just in the evolution of C4 plants. All SiGRAS genes differ in genetic structure, while members of the same subfamily have similar genetic structures. This further supports that SiGRAS genes in the common taxa share a common evolutionary origin and molecular function, making this an effective and practical method for predicting unknown protein functions [42].

\section{Expression patterns and functional prediction of the SiGRAS genes}

SiGRASO7 showed the highest expression in the flag leaves (Fig. 6), which was consistent with the expression pattern of the homologous gene AT4G37650. AT4G37650 $(A t S H R)$ may play a key role during the visible and flowering stages of leaves and the mature plant embryo stage in A. thaliana [64]. The transcription levels of SiGRASO4 and the homologous gene AT5G48150 were both high in the stems and leaves, and the AT5G48150 is required for maintenance of shoot apical meristem and the youngest primordia in A thaliana [65]. SiGRAS13, a member of the DELLA subfamily, also demonstrated higher expression during the fruit-filling stage, suggesting an important role in the development of fruits of foxtail millet. SiGRASO5 of the SCR subfamily was highly expressed in fruit, and the SHR-SCR-SCL23 module plays a key role in the formation of endodermis in A. thaliana [66]. However, specific functions need to be analyzed through in-depth 
experiments. Simultaneously, we also found that most SiGRAS members genes were expressed at higher levels in the middle filling stage (25 DPA). This finding is different from that shown by dicotyledons buckwheat [42] and castor beans [67], which indicates that these genes may be involved in middle fruit development.

The Gibberellin could be detected in the whole fruit development stage of foxtail millet (Fig. 7), which gradually decreased from 18DPA (48.92 $\mathrm{ng} \cdot \mathrm{g}^{-1}$ ) to 32DPA $\left(18.98 \mathrm{ng} \cdot \mathrm{g}^{-1}\right)$. Therefore, we hypothesized that gibberellin is produced in young fruits of foxtail millet immediately after fertilization to promote the fruit initiation process [68]. Comparing the members of the DELLA subfamily (SiGRAS13, SiGRAS25, and SiGRAS48), the expression of SiDELLAs during middle fruit development was significantly higher than that at the early (18 DPA) and late (32 DPA) stages. Therefore, we hypothesized that DELLA genes may play a role during the middle development stages of fruits. Paclobutrazol, a triazole plant growth regulator, regulates plant tissue and fruit development by inhibiting GA biosynthesis through the regulation of DELLA transcription [43]. The plant height was significantly reduced at the grain filling stage in the paclobutrazol treatment group compared to that in the mock group. Similarly, in wheat, plant height and stem length were decreased after treatment with paclobutrazol. At the same time, the stem diameter, stem plumpness, and basal internode wall thickness were significantly increased in the paclobutrazol treatment group, leading to higher stem strength and higher lodging resistance index (CLRI) [69]. Then, the expression levels of the DELLA subfamily (SiGRAS13, SiGRAS25, and SiGRAS48) in the paclobutrazol treatment group were further analysed in S. italica (Fig. 7B). The expression patterns of all DELLA genes were significantly changed in the paclobutrazol treatment group compared to that in the mock group, especially in the early filling stage. The expression levels of SiGRAS25 and SiGRAS48 changed significantly at $18 \mathrm{DPA}$, which indicated that they may be sensitive in the early stage. After paclobutrazol treatment, the expression level of SiGRAS13 expression was significantly increased throughout fruit development. Meanwhile, the sensitivity of SiGRAS13 to paclobutrazol treatment was higher than that of SiGRAS25 and SiGRAS48. Therefore, we speculate that SiGRAS13 may has potential value in the breeding of S. italica. We found that paclobutrazol had significant inhibitory effects on gibberellin synthesis, especially at the early filling stage (18DPA). We hypothesized that the expression patterns of DELLA members may be influenced by the downregulation of gibberellin (Fig. 7). At the same time, different DELLA genes may have different responses to gibberellin, most of which change significantly at the early filling stage, whereas some members have longterm responses (SiGRAS13). We hypothesized that the decrease of GA content relieved the inhibition of DELLA protein expression, and the plant height of foxtail millet was significantly inhibited. However, the application of paclobutrazol increased the 1000-grain weight of foxtail millet, which may be due to the increased accumulation of photoassimilate products [70].

As a drought-tolerant crop, foxtail millet may regulate its adaptation to the environment through complex endogenous networks and transcriptional signals, and similar conclusions have also been reached for poplar [32], sorghum [43], and Tartary buckwheat [42]. Previous studies have found that some members of the same subfamily with the same motif may have similar physiological functions [43]. Therefore, we can further speculate the function of SiGRAS genes, which needs to be further verified in experiments. AT5G41920 and SiGRASO5 both belonged to the SCR subfamily, had similar motif compositions, and were expressed preferentially under dehydration stress in A. thaliana [71]. Similarly, the expression of SiGRASO5 was significantly upregulated in the roots under PEG stress, which may enhance the adaptability of foxtail millet to the environment in a similar pattern. SiGRAS04 was classified under the PAT1 subfamily, and its expression was increased in the roots, stems, and leaves when under stress from $\mathrm{NaCl}$, heat, cold, and flooding. VaPAT1, a GRAS gene from Vitis amurensis, acts as a stress-induced GRAS gene and enhances cold, drought, and salt tolerance of transgenic Arabidopsis by regulating the expression of a series of stress-related genes [72]. Interestingly, AT1G50600, AT2G04890, AT4G17230, and AT5G48150, which belong to the PAT1 subfamily, are all involved in the optical signaling pathway and are highly expressed in the leaves [26]. Furthermore, SiGRAS04 had the same motifs and gene structures as these genes. Under dark conditions, SiGRAS04 expression was rapidly and significantly upregulated in the leaves and stems, which may help regulate photosynthesis and respiratory balance; however, this needs to be further verified by future experiments. SiGRAS47 responded significantly to different abiotic stresses in roots, and its expression was increased in $24 \mathrm{~h}$ during the seven stresses (acid, $\mathrm{NaCl}$, heat, cold, flooding, and darkness). PeSCL7, a member of the SCL subfamily in poplars, enhances drought and salt resistance at the Arabidopsis seedling stage by promoting root development and reducing water loss rate [32]. SiGRAS13, SiGRAS25, and SiGRAS48 belonged to the DELLA subfamily and were involved in almost all of the abiotic stress responses, especially SiGRAS13 and SiGRAS25. Thus, we can assume that DELLA protein mediated gibberellin signalling plays an important role in a variety of abiotic stress responses in foxtail millet, 
and different DELLA members may play regulatory roles to different degrees. In A. thaliana, the high expression level of DELLA expression can improve the activity of reactive oxygen species (ROS), protect cells from ROS damage under abiotic stress, and enhance the survival ability of plants [73]. There were significant differences in gene expression patterns under different subfamilies, nevertheless, the correlated heat maps suggested that some members may still be co-expressed (Fig. S6), such as SiGRASO5 with SiGRAS07. In Arabidopsis, SHR may interact with $S C R$ to form the SHR-SCR heterodimer through conserved GRAS domains [74], which are regarded as central regulators in the radial patterning in the roots [75]. These results suggested that GRAS gene family may regulate the tissue development process and abiotic stress response in foxtail millet, which needs further experimental verification.

\section{Conclusion}

We first identified and analyzed the genome-wide SiGRAS gene family in S. italica. 57 SiGRAS genes were distributed on eight chromosomes and divided into 13 subfamilies. Furthermore, we found that segment duplications and tandem duplications contributed to the expansion of the SiGRAS gene family, and segment duplication may have a more important contribution. Multiple sequence alignment and gene structure analysis showed that most of the SiGRAS genes lacked introns, which indicated that SiGRAS genes were conserved to some extent. In addition, we analysed the expression of 15 SiGRAS genes in different tissues (root, stem, leaf, and fruit) and fruit development stages and under eight different forms of abiotic stress. The relationship among DELLA genes, gibberellin content and fruit development in foxtail millet were further investigated. Paclobutrazol treatment significantly down-regulated plant height and gibberellin content, but increased grain weight during whole-grain development. In addition, the expression level of SiGRAS13 expression was upregulated after paclobutrazol treatment in S. bicolor; and SiGRAS25 was sensitive to the eight abiotic stresses. These findings may be valuable considerations when breeding S. italica.

\section{Methods}

\section{Gene identification}

The entire Foxtail millet genome was downloaded from the Ensembl Genomes website (http://ensemblgenomes. org/). Foxtail millet GRAS sequences were obtained through two BLASTP methods [76, 77]. Firstly, the candidate GRAS proteins of foxtail millet were authenticated by a BLASTp search. Second, we downloaded the hidden Markov model (HMM) file corresponding to the GRAS domain (PF03514) from the Pfam protein family database (http://pfam.sanger.ac.uk/). The GRAS protein sequences were retrieved from the foxtail millet genomic database using HMMER3.0 with a cutoff of 0.01 (http:// plants.ensembl. org/hmmer/index.html) [78]. The existence of the GRAS core sequences was confirmed by the PFAM and SMART programs (http://smart.embl-heide lberg.de/) [79, 80]. Finally, 57 SiGRAS genes were identified in the foxtail millet genome. Then, 57 SiGRAS proteins were used as initial queries in the NCBI protein database (https://blast.ncbi.nlm.nih.gov/Blast.cgi? PROGRAM = blastp\&PAGE_TYPE $=$ BlastSearch\&LINK LOC = blasthome) using BLASTp to verify the GRAS proteins. In addition, ExPasy (http://web.expasy.org/ protparam/) was used to identify the basic features of the trihelix proteins of the GRAS genes of $S$. italica were identified: the sequence length, isoelectric point (pi), molecular weight (mws), and subcellular localization.

\section{GRAS structure}

Based on the default parameters of ClustalW, the domain sequences of the characterized GRAS proteins of $A$. thaliana and rice were used to create multiple protein sequence alignments with the SiGRAS domain sequences of different subfamilies [81]. Then GeneDoc software and Mega7.0 were used to manually adjust the amino acid sequences of the GRAS domain in different subfamilies. Then, the conserved motif and structural differences of 57 SiGRAS proteins were analyzed $[55,82]$. The exonintron organization of GRAS genes of foxtail millet were determined by comparing predicted coding sequences with their corresponding full-length sequences using the online program Gene Structure Display Server (GSDS: http://gsds.cbi.pku.edu.cn) [83]. The conserved motifs in the identified SiGRAS proteins were identified using the MEME online program (http://meme.nbcr.net/meme/ intro.html) [82]. The optimized parameters of motif width were employed as the following:the maximum number of motifs was 10, and the optimum width was 6 to 200 residues [43]. In addition, the software PlantCARE was used to predict the cis-acting elements of $57 \mathrm{SiGRAS}$ genes in the upstream $2000 \mathrm{bp}$ range (http://bioinforma tics.psb.ugent.be/webtools/plantcare/html/?tdsou rcetag=s_pcqq_aiomsg).

\section{Chromosomal distribution and gene duplication}

All SiGRAS genes were mapped to S. italica chromosomes based on physical location information. The Circos program was used to process the chromosomal location information of the SiGRAS genes [82]. Multiple Collinearity Scanning toolkit (MCScanX) was adopted to analyze the SiGRAS gene duplication events, with the default parameters [84]. The homology of the GRAS genes between $S$. italica and the other six plants (A. thaliana, 
F. tataricum, S. lycopersicum, B. distachyon, O. sativa subsp. indica, and $Z$. mays) were analyzed using Dual Synteny Plotter (https://github.com/CJ-Chen/TBtools). The Tajima's D Neutrality Test program of Mega7.0 software was used to further analyze the evolutionary constraints acting on SiGRAS genes [43].

\section{Phylogenetic analysis and classification of SiGRAS gene family}

According to the classification of GRAS proteins of Arabidopsis, 57 GRAS proteins of S. italica were divided into several groups. In MEGA 7.0, the NJ tree was constructed used the Jukes-Cantor model. The phylogenetic tree was constructed with a bootstrap value of 1000 and assigned with Geneious R11 with BLOSUM62 cost matrix. In addition, the full-length amino acid sequences of the characteristic GRAS proteins derived from $A$. thaliana, F. tataricum, S. lycopersicum, B. distachyon, $O$. sativa subsp. indica, and Z. mays (Table S1) combined with the newly identified SiGRAS were used for phylogenetic analysis (UniProt: https://www.uniprot.org/).

\section{Plant materials, growth conditions, paclobutrazol treatment, and abiotic stress in S. italica}

The foxtail millet accessions (Yugu 1) was widely cultivated in northern China and obtained from Prof. Cheng Jianping of Guizhou University. In 2020, 'Yugu 1' was planted in the greenhouse of the experimental base located at the farm at Guizhou University. Foxtail millet plants were grown in pots filled with soil and vermiculite (1:1) in a growth room with a $16 \mathrm{~h} / 25^{\circ} \mathrm{C}$ day and $8 \mathrm{~h} / 20^{\circ} \mathrm{C}$ night regime, with a relative humidity of $75 \%$. We collected the five plants with good growth and similar growth conditions, respectively. The samples included the flag leaves, third leaves, roots, stems, fruits in the discoloration stage, and fruits and glumes in the three developmental stages (18 DPA, green fruit stage; 25 DPA, discoloration stage; and 32 DPA, initial maturity stage). In addition, the expression patterns of 15 SiGRAS genes under different stresses were further analysed. Foxtail millet plants at the seedling stage (28 days) were selected for the abiotic stress treatments, which included acid (HCL $0.1 \mathrm{~mol} / \mathrm{L})$, alkali $(\mathrm{NaOH} 0.2 \mathrm{~mol} / \mathrm{L})$, salt $(5 \%$ $\mathrm{NaCl}$ ), flooding (whole plant), drought (30\% PEG6000), darkness (complete shading), heat $\left(40^{\circ} \mathrm{C}\right)$ and cold $\left(4^{\circ} \mathrm{C}\right)$. Five replicates were collected from each stress treatment, and the expression levels were analyzed at $0 \mathrm{~h}, 2 \mathrm{~h}$, and $24 \mathrm{~h}$, respectively. In addition, 'Yugu 1' materials with similar growth statuses were selected and sprayed with $50 \mathrm{~mL}$ paclobutrazol $\left(250 \mathrm{mg} \cdot \mathrm{L}^{-1}\right)$ during the germination period. The controls (mock) were sprayed with the same amount of water. The 1000-grain weight, plant height, gibberellin content, and gene expression level of
DELLA subfamily of foxtail millet were further analyzed among control and paclobutrazol treatment at 18, 25, and 32 DPA after pollination. The samples were collected quickly put into liquid nitrogen, and stored at $-80^{\circ} \mathrm{C}$ for subsequent analysis. Each sampling and stress treatment had five biological replicates. The samples were used for quantitative PCR (qPCR) with at least three technical repeats.

\section{Total RNA extraction, CDNA reverse transcription, and $\mathrm{qRT}$-PCR analysis}

The cDNA was produced with a $1 \mathrm{mg}$ RNA sample using a PrimeScript RT Reagent Kit with gDNA Eraser (TaKaRa) and SYBR Premix Ex Taq II (TaKaRa) [85]. Total RNA was extracted using the RNA out Kit (TaKaRa) and treated with RNase-free DNase I to remove trace amounts of DNA. Gene-expression analysis of the selected genes was performed by qPCR, and the primers were designed using Primer 5.0 software (Table S9). We used the actin gene (Si001873m.g) as an internal control, which was stably expressed at each growth stage in almost all tissues. The experimental data were calculated according to the $2^{-(\Delta \Delta C T)}$ method [86].

\section{Endogenous GA analysis}

With reference to the method of Fan et al. [43], the GA content in foxtail millet fruits was determined. A fresh tissue sample of about $1 \mathrm{~g}$ fruit was collected and ground in liquid nitrogen. Fifty millilitres of $80 \%$ ethanol were added to the ground powder, which was then used for ultrasonic extraction three times for $1 \mathrm{~h}$ each time. The supernatant was concentrated once at a low temperature, and after mixing with water, an equal volume of $\mathrm{n}$-butanol was added for extraction for $1 \mathrm{~h}$. Finally, the n-butanol layer was dried under a stream of nitrogen $\left(\mathrm{N}_{2}\right)$. Ten milligrams of the dried sample were accurately weighed and dissolved in $5 \mathrm{~mL}$ methanol. The dissolved solution was filtered using a $0.22 \mu \mathrm{m}$ microporous membrane, and LC/MS was conducted for content detection.

\section{Statistical analysis}

We processed and analyzed all the above data with variance analysis with JMP6.0 software (SAS Institute), and the means were compared by the least significant difference test (LSD) at significance levels of 0.05 and 0.01 . The histogram was drawn using the Origin 2016 (OriginLab Corporation, Northampton, Massachusetts, USA). The correlation coefficients of the SiGRAS genes were defined using Sigmaplot 12.0 software based on the Pearson correlation program. The correlation coefficient was defined as significant at $P<0.05$. 


\section{Abbreviations}

At: Arabidopsis thaliana; Os: Oryza sativa; AtGRAS: Arabidopsis thaliana GRAS; OsGRAS: Oryza sativa GRAS; SiGRAS: Setaria italica GRAS; qRT-PCR: Quantitative real-time polymerase chain reaction; TF: Transcription factor; CDS: Coding sequence; HMM: Hidden Markov Model; pl: Isoelectric point; BLAST: Basic local alignment search tool; LHR I: Leucine-heptad repeat I; LHR II: Leucine-heptad repeat II; SCR: SCARECROW.

\section{Supplementary Information}

The online version contains supplementary material available at https://doi. org/10.1186/s12870-021-03277-y.

Additional file 1 : Table S1. List of the 57 S. italica GRAS genes identified in this study.

Additional file $\mathbf{2}$ : Table S2. Subfamilies and protein sequences of Arabidopsis and rice.

Additional file 3 : Table S3. Analysis and distribution of the conserved motifs of GRAS proteins.

Additional file 4 : Table S4. Cis-regulatory elements in the promoter region of GRAS genes.

Additional file $\mathbf{5}$ : Table S5. Tandem duplication events of S. italica GRAS genes.

Additional file $\mathbf{6}$ : Table S6. The 12 pairs of segmental duplicates in S. italica GRAS genes.

Additional file 7 : Table S7. One-to-one orthologous relationships between S. italica and other plants.

Additional file $\mathbf{8}$ : Table S8. Results of Tajima's D neutrality test.

Additional file 9 : Table S9. Primer sequences for qRT-PCR.

Additional file 10 : Figure S1. Multiple sequence alignment of the GRAS domains of the members of 13 phylogenetic subfamilies of the S. italica GRAS protein family. The scheme at the top depicts the locations and boundaries of the LHR I, VHIID, LHR II, PFYRE, and SAW regions within the GRAS domain.

Additional file 11 : Figure S2. Phylogenetic relationships and motif compositions of the S. italica GRAS proteins with six different plant species (A. thaliana, F. tataricum, S. lycopersicum, B. distachyon, O. sativa subsp. indica, and Z. mays). Outer panel: Unrooted phylogenetic tree constructed using Geneious R11 with the NJ method. Innermost panel: Distribution of the conserved motifs in GRAS proteins. The differently colored boxes represent different motifs and their positions in each GRAS protein sequence. The sequence information for each motif is provided in Additional file 3 : Table S3.

Additional file 12 : Figure S3. Expression patterns of 15 S. italica GRAS genes were examined during different fruit development stages: 18 DPA (early filling stage), 25 DPA (middle filling stage), and 32 DPA (initial maturity stage).

Additional file 13 : Figure S4. The correlations 15 S. italica GRAS genes in several plant organs. Positive number: positively correlated; negative number: negatively correlated. The correlation coefficients of SiGRAS genes were defined by software Sigmaplot 12.0 based on Pearson correlation program. The correlation coefficient is defined as significant correlation with $P$-value lower than 0.05

Additional file 14 : Figure S5. Expression of 15 S. italica GRAS genes under different abiotic stresses (acid, alkali, PEG, NaCl, heat, cold, flooding and dark treatments) at the seedling stage. Error bars were obtained from three measurements. Lowercase letters above the bars indicate significant differences ( $a=0.05, L S D)$ among the treatments.

Additional file 15 : Figure S6. The correlations 15S. italica GRAS genes in several abiotic stresses.

\section{Acknowledgements}

We thank all of the colleagues in our laboratory for providing useful discussions and technical assistance. We are very grateful to the editor and reviewers for critically evaluating the manuscript and providing constructive comments for its improvement.

\section{Authors' contributions}

YF planned and designed the research and analyzed the data. YF and XW wrote the manuscript. DL, HY, and LF studied gene expression using $\mathrm{QPCR}$. DL identified the S. italica bHLH gene family and analyzed its gene structure. $\mathrm{LL}$ and $\mathrm{KN}$ studied chromosome distribution, gene duplication, and syntenic analysis of the S. italica GRAS genes. LC and DX analyzed the evolutionary relationship between GRAS genes in several different species. JR and JY revised the manuscript. JC supervised the study. All authors read and approved the final manuscript.

\section{Funding}

This research was supported by the National Science Foundation of China (31560578, Cheng JP, http://www.nsfc.gov.cn), Sichuan International Science and Technology Cooperation and Exchange Research and Development Project (2018HH0116, Yan J, http://kjt.sc.gov.cn), Guizhou Science and Technology Support Project (No. 20201Y125).

\section{Availability of data and materials}

The entire Setaria italica genome sequence information was from the Ensembl Genomes website (http://ensemblgenomes.org/). The Setaria italica materials (Yugu 1) used in the experiment were supplied by Prof. Cheng Jianping of Guizhou University. The datasets supporting the conclusions of this article are included in the article and its Additional files.

\section{Declarations}

\section{Ethics approval and consent to participate}

This article does not contain any studies with human participants or animals performed by the authors. These methods were carried out in accordance with relevant guidelines and regulations. We confirm that all experimental protocols were approved by Guizhou University.

\section{Consent for publication}

Not Applicable.

\section{Competing interests}

The authors declare that they have no competing interests.

\section{Author details}

${ }^{1}$ College of Agriculture, Guizhou University, Guiyang 550025, People's Republic of China. ${ }^{2}$ School of Food and Biological engineering, Chengdu University, Chengdu 610106, People's Republic of China. ${ }^{3}$ Guizhou provincial Center For Disease Control And Prevention, Guiyang 550025, People's Republic of China. ${ }^{4}$ Chengdu Institute of Food Inspection, Chengdu 610030, People's Republic of China. ${ }^{5}$ Henan university of technology, Zhengzhou 450001, People's Republic of China. ${ }^{6}$ Department of Nursing, Sichuan Tianyi College, Mianzhu 618200, People's Republic of China.

Received: 21 July 2021 Accepted: 18 October 2021

Published online: 03 November 2021

\section{References}

1. Sun $X$, Jones WT, Rikkerink EH. GRAS proteins: the versatile roles of intrinsically disordered proteins in plant signalling. Biochem J. 2012;442(1):112. https://doi.org/10.1042/BJ20111766.

2. Peng J, Carol P, Richards DE, King KE, Cowling RJ, Murphy GP, et al. The Arabidopsis GAl gene defines a signaling pathway that negatively regulates gibberellin responses. Genes Dev. 1997;11(23):3194-205.

3. Silverstone AL, Ciampaglio CN, Sun T. The Arabidopsis RGA gene encodes a transcriptional regulator repressing the gibberellin signal transduction pathway. Plant Cell. 1998;10(2):155-69. 
4. Di Laurenzio L, Wysocka-Diller J, Malamy JE, Pysh L, Helariutta Y, Freshour $G$, et al. The SCARECROW gene regulates an asymmetric cell division that is essential for generating the radial organization of the Arabidopsis root. Cell. 1996;86(3):423-33.

5. Sun X, Xue B, Jones WT, Rikkerink E, Dunker AK, Uversky VN. A functionally required unfoldome from the plant kingdom: intrinsically disordered $\mathrm{N}$-terminal domains of GRAS proteins are involved in molecular recognition during plant development. Plant Mol Biol. 2011;77(3):205-23.

6. Blanco-Touriñán $\mathrm{N}$, Legris $\mathrm{M}$, Minguet EG, Costigliolo-Rojas $C$, Nohales MA, Iniesto E, García-León M, Pacín M, Heucken N, Blomeier T, Locascio A, Černý M, Esteve-Bruna D, Díez-Díaz M, Brzobohatý B, Frerigmann H, Zurbriggen MD, Kay SA, Rubio V, Blázquez MA, Casal JJ, Alabadí D. COP1 destabilizes DELLA proteins in Arabidopsis. Proc Natl Acad Sci U S A. 2020;117(24):13792-9. https://doi.org/10.1073/pnas.1907969117.

7. Pysh LD, Wysocka-Diller JW, Camilleri C, Bouchez D, Benfey PN. The GRAS gene family in Arabidopsis: sequence characterization and basic expression analysis of the SCARECROW-LIKE genes. Plant J. 1999;18(1):111-9. https://doi.org/10.1046/.1365-313x.1999.00431.x.

8. Richards DE, Peng J, Harberd NP. Plant GRAS and metazoan STATs: one family? Bioessays. 2000;22(6):573-7. https://doi.org/10.1002/(SICI)15211878(200006)22:6<573::AID-BIES10>3.0.CO;2-H.

9. Dill A, Jung HS, Sun TP. The DELLA motif is essential for gibberellin-induced degradation of RGA. Proc Natl Acad Sci U S A. 2001;98(24):14162-7. https://doi.org/10.1073/pnas.251534098.

10. Itoh H, Ueguchi-Tanaka M, Sato Y, Ashikari M, Matsuoka M. The gibberellin signaling pathway is regulated by the appearance and disappearance of SLENDER RICE1 in nuclei. Plant Cell. 2002;14(1):57-70. https://doi.org/10. 1105/tpc.010319.

11. Heery DM, Kalkhoven E, Hoare S, Parker MG. A signature motif in transcriptional co-activators mediates binding to nuclear receptors. Nature. 1997;387(6634):733-6. https://doi.org/10.1038/42750.

12. Hirsch S, Kim J, Muñoz A, Heckmann AB, Downie JA, Oldroyd GE. GRAS proteins form a DNA binding complex to induce gene expression during nodulation signaling in Medicago truncatula. Plant Cell. 2009;21(2):54557. https://doi.org/10.1105/tpc.108.064501.

13. De Lucas $M$, Davière JM, Rodríguez-Falcón M, Pontin M, Iglesias-Pedraz JM, Lorrain S, Fankhauser C, Blázquez MA, Titarenko E, Prat S. A molecular framework for light and gibberellin control of cell elongation. Nature. 2008:451 (7177):480-4. https://doi.org/10.1038/nature06520.

14. Hou X, Lee LY, Xia K, Yan Y, Yu H. DELLAs modulate jasmonate signaling via competitive binding to JAZs. Dev Cell. 2010;19(6):884-94. https://doi.org/ 10.1016/j.devcel.2010.10.024.

15. Tian C, Wan P, Sun S, Li J, Chen M. Genome-wide analysis of the GRAS gene family in rice and Arabidopsis. Plant Mol Biol. 2004;54(4):519-32. https://doi.org/10.1023/B:PLAN.0000038256.89809.57.

16. Cenci A, Rouard M. Evolutionary analyses of GRAS transcription factors in angiosperms. Front Plant Sci. 2017;8:273. https://doi.org/10.3389/fpls. 2017.00273.

17. Liu X, Widmer A. Genome-wide comparative analysis of the GRAS gene family in Populus, Arabidopsis and Rice. Plant Mol Biol Report. 2014;32(6):1129-45.

18. Heo JO, Chang KS, Kim IA, Lee MH, Lee SA, Song SK, Lee MM, Lim J. Funneling of gibberellin signaling by the GRAS transcription regulator scarecrow-like 3 in the Arabidopsis root. Proc Natl Acad Sci U S A. 2011;108(5):2166-71. https://doi.org/10.1073/pnas.1012215108.

19. Zhang ZL, Ogawa M, Fleet CM, Zentella R, Hu J, Heo JO, Lim J, Kamiya Y, Yamaguchi S, Sun TP. Scarecrow-like 3 promotes gibberellin signaling by antagonizing master growth repressor DELLA in Arabidopsis. Proc Natl Acad Sci U S A. 2011;108(5):2160-5. https://doi.org/10.1073/pnas.10122 32108.

20. Hakoshima T. Structural basis of the specific interactions of GRAS family proteins. FEBS Lett. 2018;592(4):489-501. https://doi.org/10.1002/18733468.12987.

21. Oldroyd GE, Long SR. Identification and characterization of nodulationsignaling pathway 2, a gene of Medicago truncatula involved in Nod actor signaling. Plant Physiol. 2003;131(3):1027-32. https://doi.org/10.1104/pp. 102.010710

22. Greb T, Clarenz O, Schafer E, Muller D, Herrero R, Schmitz G, Theres K. Molecular analysis of the LATERAL SUPPRESSOR gene in Arabidopsis reveals a conserved control mechanism for axillary meristem formation. Genes Dev. 2003;17(9):1175-87. https://doi.org/10.1101/gad.260703.
23. Scheres B, Benfey PN. Asymmetric cell division in plants. Annu Rev Plant Physiol Plant Mol Biol. 1999;50:505-37. https://doi.org/10.1146/annurev. arplant.50.1.505.

24. Bolle C, Koncz C, Chua NH. PAT1, a new member of the GRAS family, is involved in phytochrome A signal transduction. Genes Dev. 2000;14(10):1269-78.

25. Li X, Qian Q, Fu Z, Wang Y, Xiong G, Zeng D, Wang X, Liu X, Teng S, Hiroshi F, Yuan M, Luo D, Han B, Li J. Control of tillering in rice. Nature. 2003:422(6932):618-21. https://doi.org/10.1038/nature01518.

26. Torres-Galea P, Huang LF, Chua NH, Bolle C. The GRAS protein SCL13 is a positive regulator of phytochrome-dependent red light signaling, but can also modulate phytochrome A responses. Mol Gen Genomics. 2006;276(1):13-30. https://doi.org/10.1007/s00438-006-0123-y.

27. Achard P, Vriezen WH, Van Der Straeten D, Harberd NP. Ethylene regulates arabidopsis development via the modulation of DELLA protein growth repressor function. Plant Cell. 2003;15(12):2816-25. https://doi.org/10. 1105/tpc.015685

28. Fuentes S, Ljung K, Sorefan K, Alvey E, Harberd NP, Østergaard L. Fruit growth in Arabidopsis occurs via DELLA-dependent and DELLA-independent gibberellin responses. Plant Cell. 2012;24(10):3982-96. https:// doi.org/10.1105/tpc.112.103192.

29. Stuurman J, Jäggi F, Kuhlemeier C. Shoot meristem maintenance is controlled by a GRAS-gene mediated signal from differentiating cells. Genes Dev. 2002;16(17):2213-8. https://doi.org/10.1101/gad.230702.

30. Morohashi K, Minami M, Takase H, Hotta Y, Hiratsuka K. Isolation and characterization of a novel GRAS gene that regulates meiosis-associated gene expression. J Biol Chem. 2003;278(23):20865-73. https://doi.org/10. 1074/jbc.M301712200.

31. Tong H, Jin Y, Liu W, Li F, Fang J, Yin Y, Qian Q, Zhu L, Chu C. DWARF AND LOW-TILLERING, a new member of the GRAS family, plays positive roles in brassinosteroid signaling in rice. Plant J. 2009;58(5):803-16. https://doi. org/10.1111/j.1365-313X.2009.03825.X.

32. Ma HS, Liang D, Shuai P, Xia XL, Yin WL. The salt- and drought-inducible poplar GRAS protein SCL7 confers salt and drought tolerance in Arabidopsis thaliana. J Exp Bot. 2010;61 (14):4011-9. https://doi.org/10.1093/ jxb/erq217.

33. Colebrook EH, Thomas SG, Phillips AL, Hedden P. The role of gibberellin signalling in plant responses to abiotic stress. J Exp Biol. 2014;217(Pt 1):67-75. https://doi.org/10.1242/jeb.089938.

34. Achard P, Gong F, Cheminant $\mathrm{S}$, Alioua M, Hedden P, Genschik P. The cold-inducible CBF1 factor-dependent signaling pathway modulates the accumulation of the growth-repressing DELLA proteins via its effect on gibberellin metabolism. Plant Cell. 2008;20(8):2117-29. https://doi.org/10. 1105/tpc.108.058941.

35. Li P, Brutnell TP. Setaria viridis and Setaria italica, model genetic systems for the Panicoid grasses. J Exp Bot. 2011;62(9):3031-7. https://doi.org/10. 1093/jxb/erro96.

36. Liu TY, Ye N, Song T, Cao Y, Gao B, Zhang D, Zhu F, Chen M, Zhang Y, Xu $W$, Zhang J. Rhizosheath formation and involvement in foxtail millet (Setaria italica) root growth under drought stress. J Integr Plant Biol. 2019;61(4):449-62. https://doi.org/10.1111/jipb.12716.

37. Huang W, Xian Z, Kang X, Tang N, Li Z. Genome-wide identification, phylogeny and expression analysis of GRAS gene family in tomato. BMC Plant Biol. 2015;15:209.

38. Grimplet J, Agudelo-Romero P, Teixeira RT, Martinez-Zapater JM, Fortes AM. Structural and functional analysis of the GRAS gene family in grapevine indicates a role of GRAS proteins in the control of development and stress responses. Front Plant Sci. 2016;7:353.

39. Fan S, Zhang D, Gao C, Zhao M, Wu H, Li Y, et al. Identification, classification, and expression analysis of GRAS gene family in Malus domestica. Front Physiol. 2017;8:253.

40. Guo Y, Wu H, Li X, Li Q, Zhao X, Duan X, et al. Identification and expression of GRAS family genes in maize (Zea mays L.). PLoS One. 2017;12(9):e0185418.

41. Zhang B, Liu J, Yang ZE, Chen EY, Zhang CJ, Zhang XY, et al. Genome-wide analysis of GRAS transcription factor gene family in Gossypium hirsutum L. BMC Genomics. 2018;19(1):348.

42. Liu M, Huang L, Ma Z, Sun W, Wu Q, Tang Z, et al. Genome-wide identification, expression analysis and functional study of the GRAS gene family in Tartary buckwheat (Fagopyrum tataricum). BMC Plant Biol. 2019;19(1):342. 
43. Fan Y, Yan J, Lai D, Yang H, Xue G, He A, Guo T, Chen L, Cheng XB, Xiang DB, Ruan J, Cheng J. Genome-wide identification, expression analysis, and functional study of the GRAS transcription factor family and its response to abiotic stress in sorghum [Sorghum bicolor (L.) Moench]. BMCGenomics. 2021;22(1):509. https://doi.org/10.1186/s12864-021-07848-z.

44. Lu X, Liu W, Xiang C, Li X, Wang Q, Wang T, et al. Genome-wide characterization of GRAS family and their potential roles in cold tolerance of cucumber (Cucumis sativus L.). Int J Mol Sci. 2020;21(11):3857.

45. Song XM, Liu TK, Duan WK, Ma QH, Ren J, Wang Z, et al. Genome-wide analysis of the GRAS gene family in Chinese cabbage (Brassica rapa ssp. pekinensis). Genomics. 2014;103(1):135-46.

46. Chen F, Hu Y, Vannozzi A, Wu KC, Cai HY, Qin Y, et al. The WRKY transcription factor family in model plants and crops. Crit Rev Plant Sci. 2018;36(5):1-25.

47. Tajima F. Statistical method for testing the neutral mutation hypothesis by DNA polymorphism. Genetics. 1989;123(3):585-95.

48. Kumar S, Stecher G, Tamura K. MEGA7: Molecular Evolutionary Genetics Analysis Version 7.0 for Bigger Datasets. Mol Biol Evol. 2016;33(7):1870-4. https://doi.org/10.1093/molbev/msw054.

49. Bennetzen JL, Schmutz J, Wang H, Percifield R, Hawkins J, Pontaroli AC, et al. Reference genome sequence of the model plant Setaria. Nat Biotechnol. 2012;30(6):555-61.

50. Zhang G, Liu X, Quan Z, Cheng S, Xu X, Pan S, et al. Genome sequence of foxtail millet (Setaria italica) provides insights into grass evolution and biofuel potential. Nat Biotechnol. 2012;30(6):549-54.

51. Sun X, Rikkerink EH, Jones WT, Uversky VN. Multifarious roles of intrinsic disorder in proteins illustrate its broad impact on plant biology. Plant Cell. 2013;25(1):38-55

52. Magadum S, Banerjee U, Murugan P, Gangapur D, Ravikesavan R. Gene duplication as a major force in evolution. J Genet. 2013;92(1):155-61. https://doi.org/10.1007/s12041-013-0212-8.

53. Long M, VanKuren NW, Chen S, Vibranovski MD. New gene evolution: little did we know. Annu Rev Genet. 2013;47:307-33. https://doi.org/10.1146/ annurev-genet-111212-133301.

54. Bolle C. The role of GRAS proteins in plant signal transduction and development. Planta. 2004;218(5):683-92. https://doi.org/10.1007/ s00425-004-1203-z.

55. Faria R, Johannesson K, Butlin RK, Westram AM. Evolving inversions. Trends Ecol Evol. 2019;34(3):239-48. https://doi.org/10.1016/j.tree.2018.12.005.

56. Brand LH, Fischer NM, Harter K, Kohlbacher O, Wanke D. Elucidating the evolutionary conserved DNA-binding specificities of WRKY transcription factors by molecular dynamics and in vitro binding assays. Nucleic Acids Res. 2013:41(21):9764-78.

57. Xie T, Chen C, Li C, Liu J, Liu C, He Y. Genome-wide investigation of WRKY gene family in pineapple: evolution and expression profiles during development and stress. BMC Genomics. 2018;19(1):490. https://doi.org/10.1186/ s12864-018-4880-X.

58. Jain M, Nijhawan A, Arora R, Agarwal P, Ray S, Sharma P, et al. F-box proteins in rice. Genome-wide analysis, classification, temporal and spatial gene expression during panicle and seed development, and regulation by light and abiotic stress. Plant Physiol. 2007;143(4):1467-83.

59. Aubourg S, Kreis M, Lecharny A. The DEAD box RNA helicase family in Arabidopsis thaliana. Nucleic Acids Res. 1999;27(2):628-36.

60. Shabalina SA, Ogurtsov AY, Spiridonov AN, et al. Distinct patterns of expression and evolution of intronless and intron-containing mammalian genes. Mol Biol Evol. 2010;27(8):1745-9.

61. Sang YM, Liu Q, Lee J, et al. Expansion of amphibian intronless interferons revises the paradigm for interferon evolution and functional diversity. Sci Rep. 2016;6:29072.

62. Jain M, Khurana P, Tyagi AK, et al. Genome - wide analysis of intronless genes in rice and Arabidopsis. Funct Integr Genomics. 2008;8(1):69-78.

63. Lu J, Wang T, Xu Z, Sun L, Zhang Q. Genome-wide analysis of the GRAS gene family in Prunus mume. Mol Gen Genomics. 2015;290(1):303-17.

64. Wang J, Andersson-Gunnerås S, Gaboreanu I, Hertzberg M, Tucker MR, Zheng B, Leśniewska J, Mellerowicz EJ, Laux T, Sandberg G, Jones B. Reduced expression of the SHORT-ROOT gene increases the rates of growth and development in hybrid poplar and Arabidopsis. PLoS One. 2011;6(12):e28878. https://doi.org/10.1371/journal.pone.0028878.

65. Laux T, Mayer KF, Berger J, Jürgens G. The WUSCHEL gene is required for shoot and floral meristem integrity in Arabidopsis. Development. 1996;122(1):87-96.
66. Yoon EK, Dhar S, Lee MH, Song JH, Lee SA, Kim G, et al. Conservation and diversification of the SHR-SCR-SCL23 regulatory network in the development of the functional endodermis in Arabidopsis shoots. Mol Plant. 2016;9(8):1197-209.

67. Xu W, Chen Z, Ahmed N, Han B, Cui Q, Liu A. Genome-wide identification, evolutionary analysis, and stress responses of the GRAS gene family in Castor beans. Int J Mol Sci. 2016;17(7):1004.

68. Gorguet B, van Heusden AW, Lindhout P. Parthenocarpic fruit development in tomato. Plant Biol (Stuttg). 2005;7(2):131-9. https://doi.org/10. 1055/s-2005-837494.

69. Kamran M, Ahmad I, Wu X, Liu T, Ding R, Han Q. Application of paclobutrazol: a strategy for inducing lodging resistance of wheat through mediation of plant height, stem physical strength, and lignin biosynthesis. Environ Sci Pollut Res Int. 2018;25(29):29366-78. https://doi.org/10.1007/s11356-018-2965-3.

70. Kumar S, Ghatty S, Satyanarayana J, Guha A, Chaitanya B, Reddy AR. Paclobutrazol treatment as a potential strategy for higher seed and oil yield in field-grown Camelina sativa L. Crantz. BMC Res Notes. 2012;5:137. https:// doi.org/10.1186/1756-0500-5-137

71. Ding Y, Liu N, Virlouvet L, Riethoven JJ, Fromm M, Avramova Z. Four distinct types of dehydration stress memory genes in Arabidopsis thaliana. BMC Plant Biol. 2013;13:229. https://doi.org/10.1186/1471-2229-13-229.

72. Yuan Y, Fang L, Karungo SK, Zhang L, Gao Y, Li S, Xin H. Overexpression of VaPAT1, a GRAS transcription factor from Vitis amurensis, confers abiotic stress tolerance in Arabidopsis. Plant Cell Rep. 2016;35(3):655-66. https://doi. org/10.1007/s00299-015-1910-x

73. Achard P, Renou JP, Berthomé R, Harberd NP, Genschik P. Plant DELLAs restrain growth and promote survival of adversity by reducing the levels of reactive oxygen species. Curr Biol. 2008;18(9):656-60. https://doi.org/10. 1016/j.cub.2008.04.034.

74. Nakajima K, Sena G, Nawy T, Benfey PN. Intercellular movement of the putative transcription factor SHR in root patterning. Nature. 2001;413(6853):30711. https://doi.org/10.1038/35095061.

75. Hirano Y, Nakagawa M, Suyama T, Murase K, Shirakawa M, Takayama S, Sun TP, Hakoshima T. Structure of the SHR-SCR heterodimer bound to the BIRD/ IDD transcriptional factor JKD. Nat Plants. 2017;3:17010. https://doi.org/10. 1038/nplants.2017.10.

76. Altschul SF, Madden TL, Schäffer AA, Zhang J, Zhang Z, Miller W, et al. Gapped BLAST and PSI-BLAST: a new generation of protein database search programs. Nucleic Acids Res. 1997;25(17):3389-402.

77. Liu M, Ma Z, Wang A, Zheng T, Huang L, Sun W, et al. Genome-wide investigation of the auxin response factor gene family in Tartary buckwheat (Fagopyrum tataricum). Int J Mol Sci. 2018;19(11):3526.

78. Finn RD, Clements J, Eddy SR. HMMER web server: interactive sequence similarity searching. Nucleic Acids Res. 2011;39(Web Server issue):W29-37.

79. Bateman A, Birney E, Durbin R, Eddy SR, Howe KL, Sonnhammer EL. The Pfam protein families database. Nucleic Acids Res. 2000;28(1):263-6.

80. Letunic I, Bork P. 20 years of the SMART protein domain annotation resource. Nucleic Acids Res. 2018:46(D1):D493-6.

81. Thompson JD, Gibson TJ, Higgins DG. Multiple sequence alignment using ClustalW and ClustalX. Curr Protoc Bioinformatics. 2002;Chapter 2:Unit 2.3. https://doi.org/10.1002/0471250953.bi0203s00.

82. Bailey TL, Boden M, Buske FA, Frith M, Grant CE, Clementi L, et al. MEME SUITE: tools for motif discovery and searching. Nucleic Acids Res. 2009:37(Web Server issue):W202-8.

83. Guo AY, Zhu QH, Chen X, Luo JC. GSDS: a gene structure display server. Yi Chuan. 2007;29(8):1023.

84. Carretero-Paulet L, Galstyan A, Roig-Villanova I, Martínez-García JF, BilbaoCastro JR, Robertson DL. Genome-wide classification and evolutionary analysis of the bHLH family of transcription factors in Arabidopsis, poplar, rice, moss, and algae. Plant Physiol. 2010;153(3):1398-412.

85. Fan Y, Yang H, Lai D, He A, Xue G, Feng L, Chen L, Cheng XB, Ruan J, Yan J, Cheng J. Genome-wide identification and expression analysis of the bHLH transcription factor family and its response to abiotic stress in sorghum [Sorghum bicolor (L.) Moench]. BMC Genomics. 2021;22(1):415. https://doi. org/10.1186/s12864-021-07652-9.

86. Livak KJ, Schmittgen TD. Analysis of relative gene expression data using real-time quantitative PCR and the 2(-Delta Delta C(T)) method. Methods. 2001;25(4):402-8.

\section{Publisher's Note}

Springer Nature remains neutral with regard to jurisdictional claims in published maps and institutional affiliations. 